\title{
Systematics of fully heavy tetraquarks
}

\author{
Xin-Zhen Weng $\odot,{ }^{1, *}$ Xiao-Lin Chen, ${ }^{1, \dagger}$ Wei-Zhen Deng $\odot,{ }^{1, *}$ and Shi-Lin Zhu ${ }^{1,2,3,8}$ \\ ${ }^{1}$ School of Physics and State Key Laboratory of Nuclear Physics and Technology, Peking University, \\ Beijing 100871, China \\ ${ }^{2}$ Center of High Energy Physics, Peking University, Beijing 100871, China \\ ${ }^{3}$ Collaborative Innovation Center of Quantum Matter, Beijing 100871, China
}

(Received 16 October 2020; accepted 10 January 2021; published 1 February 2021)

\begin{abstract}
In this work, we systematically study the mass spectrum of the fully heavy tetraquark in an extended chromomagnetic model, which includes both color and chromomagnetic interactions. Numerical results indicate that the energy level is mainly determined by the color interaction, which favors the color-sextet $\left|(Q Q)^{6_{c}}(\bar{Q} \bar{Q})^{\overline{6}_{c}}\right\rangle$ configuration over the color-triplet $\left|(Q Q)^{\overline{3}_{c}}(\bar{Q} \bar{Q})^{3_{c}}\right\rangle$ one. The chromomagnetic interaction mixes the two color configurations and gives small splitting. The ground state is always dominated by the color-sextet configuration. We find no stable state below the lowest heavy quarkonium pair thresholds. Most states may be wide since they have at least one $S$-wave decay channel into two $S$-wave mesons. One possible narrow state is the $1^{+} b b \bar{b} \bar{c}$ state with a mass $15719.1 \mathrm{MeV}$. It is just above the $\eta_{b} \bar{B}_{c}$ threshold. But this channel is forbidden because of the conservation of the angular momentum and parity.
\end{abstract}

DOI: 10.1103/PhysRevD.103.034001

\section{INTRODUCTION}

In the quark model [1,2], a normal hadron is composed of quark-antiquark pair (meson) or three quarks (baryons). The exotic states consisting of more than three quarks have not been observed until this century. In 2003, the Belle Collaboration measured the exclusive $B^{ \pm} \rightarrow K^{ \pm} \pi^{+} \pi^{-} J / \psi$ decays, and observed the first charmoniumlike state $X(3872)$ [3], whose quantum number was later determined to be $I^{G} J^{P C}=0^{+} 1^{++}[4]$. Since then, lots of charmoniumlike and bottomoniumlike states are found, such as the $Y(4260)$ [5], $Z_{c}(3900)$ [6,7], $Z_{b}(10610)$, and $Z_{b}(10650)$ [8] states. Various theoretical interpretations, such as the molecule [9-11], the compact tetraquark [12,13], the hybrid meson [14,15], etc., have been proposed to explain their nature. More details can be found in Refs. [16-23] and references therein.

Another interesting structure is the fully heavy tetraquarks. In 2016, the CMS collaboration reported the first observation of the $\Upsilon(1 S)$ pair production in $p p$ collisions

\footnotetext{
*xzhweng@pku.edu.cn

†chenxl@pku.edu.cn

*dwz@pku.edu.cn

§husl@pku.edu.cn
}

Published by the American Physical Society under the terms of the Creative Commons Attribution 4.0 International license. Further distribution of this work must maintain attribution to the author(s) and the published article's title, journal citation, and DOI. Funded by SCOAP . at $\sqrt{s}=8 \mathrm{TeV}$ [24]. They found an exotic structure in $\mu^{+} \mu^{-} \mu^{+} \mu^{-}$channel with a global significance of $3.6 \sigma$. Its invariant mass is $18.4 \pm 0.1$ (stat) \pm 0.2 (syst) $\mathrm{GeV}$ [25]. Two years later, the LHCb collaboration searched for the $X_{b \bar{b} b \bar{b}}$ in the $\Upsilon(1 S) \mu^{+} \mu^{-}$final state, but they did not see any significant excess in the range $17.5-20.0 \mathrm{GeV}$ [26]. Last year, the $\mathrm{A}_{N} \mathrm{DY}$ collaboration investigated the dijet mass in $\mathrm{Cu}+\mathrm{Au}$ collisions, and found a peak at $M=$ $18.12 \pm 0.15$ (stat) \pm 0.6 (syst) $\mathrm{GeV}$, which is possibly an all- $b$ tetraquark [27]. In the full-charm sector, the charmonium pair production had also been observed by the NA3 [28,29], D0 [30], LHCb [31] and Belle [32] collaborations. Very recently, the $\mathrm{LHCb}$ collaboration observed a narrow structure and a wide structure in the $J / \psi$-pair invariant mass spectrum in the range of $6.2-7.2 \mathrm{GeV}$, which could be all-charm hadrons [33].

Theoretically, the fully heavy tetraquarks have been studied with various methods, such as the potential quark model [34-47], the QCD sum rules [48-52], the covariant Bethe-Salpeter equations [53] and the lattice QCD [54]. In Ref. [37], Debastiani and Navarra found that the lowest $S$-wave $c c \bar{c} \bar{c}$ tetraquarks might be below the dicharmonium thresholds. Anwar et al. [55] studied the $b b \bar{b} \bar{b}$ tetraquarks with a nonrelativistic effective field theory (NREFT) at the leading order (LO) and a relativized diquark model. The ground state masses in the two approaches are $18.72 \pm 0.02 \mathrm{GeV}$ and $18.75 \mathrm{GeV}$ respectively, which indicates the existence of a $b b \bar{b} \bar{b}$ bound state below the $\eta_{b} \eta_{b}$ threshold. However, many other studies suggest that the ground state of fully heavy tetraquarks is 
above the di-meson threshold. For example, Wang et al. [42] used two quark models with different potentials to study the $Q Q \bar{Q}^{\prime} \bar{Q}^{\prime}$ tetraquarks. They found that the $Q Q \bar{Q}^{\prime} \bar{Q}^{\prime}$ tetraquarks are always above the $\eta_{Q} \eta_{Q} / B_{c} B_{c}$ thresholds and no bound tetraquark states exist. In Ref. [54], Hughes et al. adopted the first-principles lattice nonrelativistic QCD methodology to study the lowest energy eigenstate of the $b b \bar{b} \bar{b}$ systems with quantum numbers $0^{++}, 1^{+-}$and $2^{++}$, and did not find any state below the lowest bottomonium-pair threshold.

In the quark model [56-60], the hadron mass can be decomposed into the quark masses, the kinetic energy and the potential interaction. Usually, the potential includes the color-independent Coulomb and confinement interactions, and the hyperfine interactions like the spin-spin, spin-orbit, and tensor terms.

When restricted to the ground state, one can use the simplified chromomagnetic model [12,36,60-64]. In this model, the quark masses, the kinetic energy and the spinindependent interaction are absorbed into the effective quark masses, and the spin-spin interaction is simplified to be the chromomagnetic interaction. The spin-orbit and tensor interactions are ignored since we only consider the $S$-wave states. This simplified model can well explain the hyperfine splittings of ordinary hadrons. However, the recent studies indicate that the one-body effective quark masses are not enough to account for all of the two-body spin-independent color interaction. For example, Karliner et al. [65] found that a color-related interaction between heavy (anti-)quark and strange (or heavy) quark should be introduced to account for the heavy meson and baryon masses. Then they used this model to predict the $\Xi_{c c}$ mass $M_{\Xi_{c c}}=3627 \pm 12 \mathrm{MeV}$, which is very close to the LHCb's measurement [66].

In Ref. [67], we generalized the chromomagnetic model by including the color interaction in the Hamiltonian. For the color-singlet hadrons, we found that the effective quark masses can be absorbed into the color interaction. With this model, we can reproduce the mass of doubly charm baryon $\Xi_{c c}$ [67] and the recently observed $P_{c}$ pentaquarks [68]. In this work, we will use the extended chromomagnetic model to study the spectrum of the $S$-wave fully heavy tetraquarks. With the wave function obtained, we can also estimate their decay properties.

This work is organized as follows. In Sec. II, we introduce the extended chromomagnetic model, and present the wave function bases of the fully heavy tetraquarks. Then we discuss the numerical results in Sec. III. In Sec. IV we give a brief summary.

\section{THE EXTENDED CHROMOMAGNETIC MODEL}

In the chromomagnetic model, the mass of the $S$-wave hadron consists of the effective quark masses and chromomagnetic $(\mathrm{CM})$ interaction $[12,23,36,64]$

$$
H=\sum_{i} m_{i}-\sum_{i<j} v_{i j} \boldsymbol{S}_{i} \cdot \boldsymbol{S}_{j} \boldsymbol{F}_{i} \cdot \boldsymbol{F}_{j}
$$

where $\boldsymbol{S}_{i}=\boldsymbol{\sigma}_{i} / 2\left(\boldsymbol{F}_{i}=\lambda_{i} / 2\right)$ is the quark spin operator (color operator). For the antiquark, $\boldsymbol{S}_{\bar{q}}=-\boldsymbol{S}_{q}^{*}$ and $\boldsymbol{F}_{\bar{q}}=-\boldsymbol{F}_{q}^{*}$. The $m_{i}$ is the $i$ th quark's (or antiquark's) effective mass. And the $v_{i j}$ is effective coupling constant

$$
v_{i j}=\frac{8 \pi}{3 m_{i} m_{j}}\left\langle\alpha_{s}(r) \delta^{3}(\boldsymbol{r})\right\rangle
$$

which depends on the constituent quark masses and the spatial wave function.

Comparing to the dynamical quark model $[58,59]$, we see that there is an additional color interaction, which contributes to the mass of the $S$-wave hadron, and is impossible to be absorbed into the effective quark masses or the chromomagnetic interaction. This is also supported by some concrete studies concerning baryons [65] and tetraquarks [69]. Here we introduce a colorelectric term into the model $[67,69]$

$$
H_{\mathrm{CE}}=-\sum_{i<j} A_{i j} \lambda_{i} \cdot \lambda_{j}
$$

Since

$$
\begin{aligned}
& \sum_{i<j}\left(m_{i}+m_{j}\right) \boldsymbol{F}_{i} \cdot \boldsymbol{F}_{j} \\
& \quad=\left(\sum_{i} m_{i} \boldsymbol{F}_{i}\right) \cdot\left(\sum_{i} \boldsymbol{F}_{i}\right)-\frac{4}{3} \sum_{i} m_{i},
\end{aligned}
$$

and the total color operator $\sum_{i} \boldsymbol{F}_{i}$ nullifies any color-singlet physical state, we can rewrite the model Hamiltonian as $[67,68]$

$$
H=-\frac{3}{4} \sum_{i<j} m_{i j} V_{i j}^{\mathrm{C}}-\sum_{i<j} v_{i j} V_{i j}^{\mathrm{CM}}
$$

where the quark pair mass parameter is

$$
m_{i j}=\left(m_{i}+m_{j}\right)+\frac{16}{3} A_{i j},
$$

and the color and CM interactions between quarks are defined as

$$
\begin{gathered}
V_{i j}^{\mathrm{C}}=\boldsymbol{F}_{i} \cdot \boldsymbol{F}_{j}, \\
V_{i j}^{\mathrm{CM}}=\boldsymbol{S}_{i} \cdot \boldsymbol{S}_{j} \boldsymbol{F}_{i} \cdot \boldsymbol{F}_{j} .
\end{gathered}
$$




\section{A. The tetraquark wave functions}

To investigate the mass spectra of the tetraquark states, we need to construct the wave functions. The total wave function is a direct product of the flavor, color, spin, and orbital wave functions. For the $S$-wave states, the orbital wave function is symmetric. Moreover, the Hamiltonian does not contain a flavor operator explicitly. Thus we first construct the color-spin wave function, and then incorporate the flavor wave function to account for the Pauli principle.

The spins of the tetraquark states can be 0,1 and 2 . In the $q q \otimes \bar{q} \bar{q}$ configuration, the possible color-spin wave functions $\left\{\alpha_{i}^{J}\right\}$ are listed as follows,

(i) $J^{P}=0^{+}$:

$$
\begin{aligned}
& \alpha_{1}^{0}=\left|\left(q_{1} q_{2}\right)_{1}^{6}\left(\bar{q}_{3} \bar{q}_{4}\right)_{1}^{\overline{6}}\right\rangle_{0}, \\
& \alpha_{2}^{0}=\left|\left(q_{1} q_{2}\right)_{0}^{6}\left(\bar{q}_{3} \bar{q}_{4}\right)_{0}^{\overline{6}}\right\rangle_{0}, \\
& \alpha_{3}^{0}=\left|\left(q_{1} q_{2}\right)_{1}^{\overline{3}}\left(\bar{q}_{3} \bar{q}_{4}\right)_{1}^{3}\right\rangle_{0}, \\
& \alpha_{4}^{0}=\left|\left(q_{1} q_{2}\right)_{0}^{\overline{3}}\left(\bar{q}_{3} \bar{q}_{4}\right)_{0}^{3}\right\rangle_{0},
\end{aligned}
$$

(ii) $J^{P}=1^{+}$:

$$
\begin{aligned}
& \alpha_{1}^{1}=\left|\left(q_{1} q_{2}\right)_{1}^{6}\left(\bar{q}_{3} \bar{q}_{4}\right)_{1}^{\overline{6}}\right\rangle_{1}, \\
& \alpha_{2}^{1}=\left|\left(q_{1} q_{2}\right)_{1}^{6}\left(\bar{q}_{3} \bar{q}_{4}\right)_{0}^{\overline{6}}\right\rangle_{1}, \\
& \alpha_{3}^{1}=\left|\left(q_{1} q_{2}\right)_{0}^{6}\left(\bar{q}_{3} \bar{q}_{4}\right)_{1}^{\overline{6}}\right\rangle_{1}, \\
& \alpha_{4}^{1}=\left|\left(q_{1} q_{2}\right)_{1}^{\overline{3}}\left(\bar{q}_{3} \bar{q}_{4}\right)_{1}^{3}\right\rangle_{1}, \\
& \alpha_{5}^{1}=\left|\left(q_{1} q_{2}\right)_{1}^{3}\left(\bar{q}_{3} \bar{q}_{4}\right)_{0}^{3}\right\rangle_{1}, \\
& \alpha_{6}^{1}=\left|\left(q_{1} q_{2}\right)_{0}^{3}\left(\bar{q}_{3} \bar{q}_{4}\right)_{1}^{3}\right\rangle_{1},
\end{aligned}
$$

(iii) $J^{P}=2^{+}$:

$$
\begin{aligned}
& \alpha_{1}^{2}=\left|\left(q_{1} q_{2}\right)_{1}^{6}\left(\bar{q}_{3} \bar{q}_{4}\right)_{1}^{\overline{6}}\right\rangle_{2}, \\
& \alpha_{2}^{2}=\left|\left(q_{1} q_{2}\right)_{1}^{\overline{3}}\left(\bar{q}_{3} \bar{q}_{4}\right)_{1}^{3}\right\rangle_{2},
\end{aligned}
$$

where the superscript $3, \overline{3}, 6$, or $\overline{6}$ denotes the color, and the subscript 0,1 , or 2 denotes the spin.

Next we consider the flavor wave function. There are three types of total wave functions when we consider the Pauli principle:

(1) Type A [Flavor $=\{c c \bar{c} \bar{c}, b b \bar{b} \bar{b}, c c \bar{b} \bar{b}\}]$ :

(a) $J^{P(C)}=0^{+(+)}$:

$$
\begin{aligned}
& \Psi_{A 1}^{0^{+(+)}}=q_{1} q_{2} \bar{q}_{3} \bar{q}_{4} \otimes \alpha_{2}^{0}, \\
& \Psi_{A 2}^{0^{+(+)}}=q_{1} q_{2} \bar{q}_{3} \bar{q}_{4} \otimes \alpha_{3}^{0},
\end{aligned}
$$

(b) $J^{P(C)}=1^{+(-)}$:

$$
\Psi_{A}^{1^{+(-)}}=q_{1} q_{2} \bar{q}_{3} \bar{q}_{4} \otimes \alpha_{4}^{1},
$$

(c) $J^{P(C)}=2^{+(+)}$:

$$
\Psi_{A}^{2^{+(+)}}=q_{1} q_{2} \bar{q}_{3} \bar{q}_{4} \otimes \alpha_{2}^{2},
$$

(2) Type B [Flavor $=\{c c \bar{c} \bar{b}, b b \bar{b} \bar{c}\}]$ :

(a) $J^{P}=0^{+}$:

$$
\begin{aligned}
& \Psi_{B 1}^{0^{+}}=q_{1} q_{2} \bar{q}_{3} \bar{q}_{4} \otimes \alpha_{2}^{0}, \\
& \Psi_{B 2}^{0^{+}}=q_{1} q_{2} \bar{q}_{3} \bar{q}_{4} \otimes \alpha_{3}^{0},
\end{aligned}
$$

(b) $J^{P}=1^{+}$:

$$
\begin{aligned}
& \Psi_{B 1}^{1^{+}}=q_{1} q_{2} \bar{q}_{3} \bar{q}_{4} \otimes \alpha_{3}^{1}, \\
& \Psi_{B 2}^{1^{+}}=q_{1} q_{2} \bar{q}_{3} \bar{q}_{4} \otimes \alpha_{4}^{1}, \\
& \Psi_{B 3}^{1^{+}}=q_{1} q_{2} \bar{q}_{3} \bar{q}_{4} \otimes \alpha_{5}^{1},
\end{aligned}
$$

(c) $J^{P}=2^{+}$:

$$
\Psi_{B}^{2^{+}}=q_{1} q_{2} \bar{q}_{3} \bar{q}_{4} \otimes \alpha_{2}^{2},
$$

(3) Type $\mathrm{C}$ [Flavor $=\{c b \bar{c} \bar{b}\}]$ :

(a) $J^{P C}=0^{++}$

$$
\begin{aligned}
& \Psi_{C 1}^{0^{++}}=q_{1} q_{2} \bar{q}_{3} \bar{q}_{4} \otimes \alpha_{1}^{0}, \\
& \Psi_{C 2}^{0^{++}}=q_{1} q_{2} \bar{q}_{3} \bar{q}_{4} \otimes \alpha_{2}^{0}, \\
& \Psi_{C 3}^{0^{++}}=q_{1} q_{2} \bar{q}_{3} \bar{q}_{4} \otimes \alpha_{3}^{0}, \\
& \Psi_{C 4}^{0^{++}}=q_{1} q_{2} \bar{q}_{3} \bar{q}_{4} \otimes \alpha_{4}^{0},
\end{aligned}
$$

(b) $J^{P C}=1^{++}$:

$$
\begin{aligned}
& \Psi_{C 1}^{1^{++}}=q_{1} q_{2} \bar{q}_{3} \bar{q}_{4} \otimes \frac{1}{\sqrt{2}}\left(\alpha_{2}^{1}+\alpha_{3}^{1}\right), \\
& \Psi_{C 2}^{1^{++}}=q_{1} q_{2} \bar{q}_{3} \bar{q}_{4} \otimes \frac{1}{\sqrt{2}}\left(\alpha_{5}^{1}+\alpha_{6}^{1}\right),
\end{aligned}
$$

(c) $J^{P C}=1^{+-}$:

$$
\begin{aligned}
& \Psi_{C 1}^{1^{+-}}=q_{1} q_{2} \bar{q}_{3} \bar{q}_{4} \otimes \alpha_{1}^{1}, \\
& \Psi_{C 2}^{1^{+-}}=q_{1} q_{2} \bar{q}_{3} \bar{q}_{4} \otimes \frac{1}{\sqrt{2}}\left(\alpha_{2}^{1}-\alpha_{3}^{1}\right), \\
& \Psi_{C 3}^{1^{+-}}=q_{1} q_{2} \bar{q}_{3} \bar{q}_{4} \otimes \alpha_{4}^{1}, \\
& \Psi_{C 4}^{1^{++-}}=q_{1} q_{2} \bar{q}_{3} \bar{q}_{4} \otimes \frac{1}{\sqrt{2}}\left(\alpha_{5}^{1}-\alpha_{6}^{1}\right),
\end{aligned}
$$

(d) $J^{P C}=2^{++}$:

$$
\begin{aligned}
& \Psi_{C 1}^{2++}=q_{1} q_{2} \bar{q}_{3} \bar{q}_{4} \otimes \alpha_{1}^{2}, \\
& \Psi_{C 2}^{2++}=q_{1} q_{2} \bar{q}_{3} \bar{q}_{4} \otimes \alpha_{2}^{2} .
\end{aligned}
$$

Diagonalizing the Hamiltonian [Eq. (5)] in these bases, we can obtain the mass spectra and eigenvectors of the fully heavy tetraquarks. 
TABLE I. Parameters of the $q \bar{q}$ and $q q$ pairs (in units of $\mathrm{MeV}$ ).

\begin{tabular}{lcccccccccc}
\hline \hline Parameter & $m_{n \bar{n}}$ & $m_{n \bar{s}}$ & $m_{s \bar{s}}$ & $m_{n \bar{c}}$ & $m_{s \bar{c}}$ & $m_{c \bar{c}}$ & $m_{n \bar{b}}$ & $m_{s \bar{b}}$ & $m_{c \bar{b}}$ & $m_{b \bar{b}}$ \\
Value & 615.95 & 794.22 & 936.40 & 1973.22 & 2076.14 & 3068.53 & 5313.35 & 5403.25 & 6322.27 & 9444.97 \\
Parameter & $v_{n \bar{n}}$ & $v_{n \bar{s}}$ & $v_{s \bar{s}}$ & $v_{n \bar{c}}$ & $v_{s \bar{c}}$ & $v_{c \bar{c}}$ & $v_{n \bar{b}}$ & $v_{s \bar{b}}$ & $v_{c \bar{b}}$ & $v_{b \bar{b}}$ \\
Value & 477.92 & 298.57 & 249.18 & 106.01 & 107.87 & 85.12 & 33.89 & 36.43 & 47.18 & 45.98 \\
Parameter & $m_{n n}$ & $m_{n s}$ & $m_{s s}$ & $m_{n c}$ & $m_{s c}$ & $m_{c c}$ & $m_{n b}$ & $m_{s b}$ & $m_{c b}$ & $m_{b b}$ \\
Value & 724.85 & 906.65 & 1049.36 & 2079.96 & 2183.68 & 3171.51 & 5412.25 & 5494.80 & 6416.07 & 9529.57 \\
Parameter & $v_{n n}$ & $v_{n s}$ & $v_{s s}$ & $v_{n c}$ & $v_{s c}$ & $v_{c c}$ & $v_{n b}$ & $v_{s b}$ & $v_{c b}$ & $v_{b b}$ \\
Value & 305.34 & 212.75 & 195.30 & 62.81 & 70.63 & 56.75 & 19.92 & 8.47 & 31.45 & 30.65 \\
\hline \hline
\end{tabular}

\section{NUMERICAL RESULTS}

\section{A. Parameters}

To obtain the masses of fully heavy tetraquarks, we need to estimate the parameters. In Ref. [67], we used the mesons to extract the parameters $m_{q \bar{q}}$ and $v_{q \bar{q}}$. Then we used the light and singly heavy baryons to extract the $m_{q q}$ and $v_{q q}$ with at most one heavy quark. Finally, we used a quark model consideration to estimate the $m_{Q Q}$ and $v_{Q Q}$. The parameters are listed in Table I. With these parameters, we can reproduce the meson and baryon masses. Especially, we obtained the $\Xi_{c c}$ baryon at $3633.3 \pm$ 9.3 MeV, which is very close to the LHCb's measurement [66]. In Ref. [68], we further used these parameters to study the hidden-charm pentaquark states, and successfully reproduced the masses of the three newly observed $P_{c}$ states, $P_{c}(4312), P_{c}(4440)$, and $P_{c}(4450)$ [70], as well as the older one, $P_{c}(4380)$ [71]. In the present work, we use the same set of parameters to estimate the mass spectrum of the ground state $Q Q \bar{Q} \bar{Q}$ tetraquarks.

\section{B. The $c c \bar{c} \bar{c}$ and $b b \bar{b} \bar{b}$ systems}

Inserting the parameters into the Hamiltonian, we can obtain the mass spectra of tetraquarks. The masses and eigenvectors of the $c c \bar{c} \bar{c}$ and $b b \bar{b} \bar{b}$ tetraquarks are listed in Table II. In the following, we will use $T\left(Q Q \bar{Q} \bar{Q}, m, J^{P C}\right)$ to denote the $Q Q \bar{Q} \bar{Q}$ tetraquarks. In both cases, the lightest states have quantum number $J^{P C}=0^{++}$. They are $T\left(c c \bar{c} \bar{c}, 6044.9,0^{++}\right)$and $T\left(b b \bar{b} \bar{b}, 18836.1,0^{++}\right)$, respectively. In Fig. 1, we plot the relative position of

TABLE II. Masses and eigenvectors of the $c c \bar{c} \bar{c}$ and $b b \bar{b} \bar{b}$ tetraquarks. The masses are all in units of $\mathrm{MeV}$.

\begin{tabular}{llcc}
\hline \hline System & $J^{P C}$ & Mass & Eigenvector \\
\hline$c c \bar{c} \bar{c}$ & $0^{++}$ & 6044.9 & $\{0.834,0.552\}$ \\
& & 6271.3 & $\{-0.552,0.834\}$ \\
& $1^{+-}$ & 6230.6 & $\{1\}$ \\
& $2^{++}$ & 6287.3 & $\{1\}$ \\
$b b \bar{b} \bar{b}$ & $0^{++}$ & 18836.1 & $\{0.903,0.431\}$ \\
& & 18981.0 & $\{-0.431,0.903\}$ \\
& $1^{+-}$ & 18969.4 & $\{1\}$ \\
& $2^{++}$ & 19000.1 & $\{1\}$ \\
\hline \hline
\end{tabular}

the $c c \bar{c} \bar{c}$ and $b b \bar{b} \bar{b}$ tetraquarks, along with meson-meson thresholds which they can decay into through quark rearrangement. From the figure, we can easily see that all states are above thresholds. Among them, the two ground states are only above the thresholds of the two pseudoscalar mesons, while the other states are all above the thresholds of two vector mesons.

Besides the masses, the eigenvectors also provide important information of the tetraquarks. The $0^{++}$states are of particular interests since they have two bases. Their color configurations are $\left|(Q Q)^{6_{c}} \otimes(\bar{Q} \bar{Q})^{\overline{6}_{c}}\right\rangle$ and $\left|(Q Q)^{\overline{3}_{c}} \otimes(\bar{Q} \bar{Q})^{3_{c}}\right\rangle$ respectively. For simplicity, we denote them as $6_{c} \otimes \overline{6}_{c}$ and $\overline{3}_{c} \otimes 3_{c}$. In the one-gluon-exchange (OGE) picture, the interaction between two quarks are attractive if they combine into a $\overline{3}_{c}$ diquark $(q q)^{\overline{3}_{c}}$, while repulsive if they combine into a $6_{c}$ diquark $(q q)^{6_{c}}$. However, the attraction between a $6_{c}$ diquark and a $\bar{\sigma}_{c}$ anti-diquark is much stronger than that between the $\overline{3}_{c} \otimes 3_{c}$ counterpart. The two competing effects make the tetraquark much more complicated compared to the ordinary hadrons [42]. In Table II, we present the eigenvectors of the tetraquark states. We see that the ground states are both dominated by the $\sigma_{c} \otimes \overline{6}_{c}$ components. More precisely, the $T\left(c c \bar{c} \bar{c}, 6044.9,0^{++}\right)$state has $69.5 \%$ of the $6_{c} \otimes \overline{6}_{c}$ component and the $T\left(b b \bar{b} \bar{b}, 18836.1,0^{++}\right)$state has $81.5 \%$. Here we illustrate the underlying dynamics as follows. We first consider the color interaction. Because of the symmetry between quarks (or antiquarks), the interaction strength between two quarks equals to that between two antiquarks, and the $Q \bar{Q}$ interactions share one strength. More precisely, we have (taking $b b \bar{b} \bar{b}$ as an example)

$$
\begin{aligned}
& \left\langle H_{\mathrm{C}}(b b \bar{b} \bar{b})\right\rangle \\
& =-\frac{3}{4}\left\langle m_{b b}\left(V_{12}^{\mathrm{C}}+V_{34}^{\mathrm{C}}\right)+m_{b \bar{b}}\left(V_{13}^{\mathrm{C}}+V_{24}^{\mathrm{C}}+V_{14}^{\mathrm{C}}+V_{23}^{\mathrm{C}}\right)\right\rangle \\
& =-\frac{3}{4}\left\langle m_{b \bar{b}} \sum_{i<j} V_{i j}^{\mathrm{C}}+2 \delta m_{b}\left(V_{12}^{\mathrm{C}}+V_{34}^{\mathrm{C}}\right)\right\rangle \\
& =2 m_{b \bar{b}}-\frac{3}{2} \delta m_{b}\left\langle V_{12}^{\mathrm{C}}+V_{34}^{\mathrm{C}}\right\rangle \\
& =2 m_{b \bar{b}}+\delta m_{b}\left(\begin{array}{cc}
-1 & 0 \\
0 & +2
\end{array}\right)
\end{aligned}
$$


6271

6231

$0^{++} 1^{+-} \quad 2^{++}$

(a) $c c \bar{c} \bar{c}$ states

(b) $b b \bar{b} \bar{b}$ states

FIG. 1. Mass spectra of the $c c \bar{c} \bar{c}$ and $b b \bar{b} \bar{b}$ tetraquark states. The dotted lines indicate various meson-meson thresholds. The masses are all in units of $\mathrm{MeV}$.

where $\delta m_{b} \equiv\left(m_{b b}-m_{b \bar{b}}\right) / 2$. In Ref. [67], we have estimated the $\delta m_{b}$ to be $42.30 \mathrm{MeV}$. Here we see that the color interaction do not mix the $\sigma_{c} \otimes \overline{6}_{c}$ and $\overline{3}_{c} \otimes 3_{c}$ configurations. Moreover, the color interaction favors the $6_{c} \otimes \overline{6}_{c}$ configuration over the $\overline{3}_{c} \otimes 3_{c}$ one. Note that these conclusions still hold if we include the internal dynamics in the quark model $[42,44]$. Similarly, the CM interaction in the two bases is

$$
\begin{aligned}
\left\langle H_{\mathrm{CM}}(b b \bar{b} \bar{b})\right\rangle & =\left(\begin{array}{cc}
\frac{1}{2} v_{b b} & \sqrt{\frac{3}{2}} v_{b \bar{b}} \\
\sqrt{\frac{3}{2}} v_{b \bar{b}} & \frac{1}{3} v_{b b}-\frac{2}{3} v_{b \bar{b}}
\end{array}\right) \\
& =v_{b \bar{b}}\left(\begin{array}{cc}
\frac{1}{3} & \sqrt{\frac{3}{2}} \\
\sqrt{\frac{3}{2}} & -\frac{4}{9}
\end{array}\right) .
\end{aligned}
$$


In the last line, we have used $v_{b b} / v_{b \bar{b}}=2 / 3$, which was estimated from the meson and baryon spectra [67] (see Table I) and also interpreted in the quark model using the Cornell or Logarithmic potential [72]. Here, $v_{b \bar{b}}=45.98 \mathrm{MeV}$. If we ignore the off-diagonal terms, we see that the CM interaction favors the $\overline{3}_{c} \otimes 3_{c}$ configuration, which is opposite to that of colorelectric interaction. However, the splitting induced by the CM interaction is much smaller than that of the colorelectric interaction $(\sim 28 \%)$, thus the net effect is that the $\sigma_{c} \otimes \bar{\sigma}_{c}$ configuration has lower mass. Similar pattern exists in the bound state of quantum electrodynamics, where the Coulomb interaction determines the energy bands, and the hyperfine interaction gives small shift of each band. Including the off-diagonal terms will push down the lower eigenstate and raise the upper eigenstate, thus the proceeding conclusion still holds. If we go to the $c c \bar{c} \bar{c}$ system, the splitting induced by the colorelectric interaction does not change much, while the CM interaction becomes larger $\left(v_{q \bar{q}} \sim 1 / m_{q} m_{\bar{q}}\right)$. The net effect is that the relative size between the off-diagonal terms and the splitting induced by diagonal terms becomes larger, which causes much stronger mixing in the $c c \bar{c} \bar{c}$ system than in the $b b \bar{b} \bar{b}$ system, as shown in Table II.

Next we consider the decay properties of the $c c \bar{c} \bar{c}$ and $b b \bar{b} \bar{b}$ tetraquarks. In the $Q \bar{Q} \otimes Q \bar{Q}$ configuration, the color wave function of the tetraquark falls into two categories: the color-singlet $\left|(Q \bar{Q})^{1_{c}}(Q \bar{Q})^{1_{c}}\right\rangle$ and the color-octet $\left|(Q \bar{Q})^{8_{c}}(Q \bar{Q})^{8_{c}}\right\rangle$. The former one can easily decay into two $S$-wave mesons (the so-called "OkuboZweig-Iizuka- (OZI-)superallowed" decays), and the latter one can only fall apart through the gluon exchange $[62,73]$. In this work, we will focus on the "OZI-superallowed" decays. We transform the eigenvectors of the tetraquark states into the $c \bar{c} \otimes c \bar{c} / b \bar{b} \otimes b \bar{b}$ configuration. The corresponding eigenvectors are listed in Tables III-IV.

TABLE III. The eigenvectors of the $c c \bar{c} \bar{c}$ tetraquarks in the $c \bar{c} \otimes c \bar{c}$ configuration. The masses are all in units of $\mathrm{MeV}$.

\begin{tabular}{lcrrrrr}
\hline \hline System & $J^{P C}$ & Mass & $\psi \psi$ & $\psi \eta_{c}$ & $\eta_{c} \psi$ & $\eta_{c} \eta_{c}$ \\
\hline$c c \bar{c} \bar{c}$ & $0^{++}$ & 6044.9 & 0.430 & & & 0.616 \\
& & 6271.3 & -0.631 & & & 0.191 \\
& $1^{+-}$ & 6230.6 & & 0.408 & 0.408 & \\
& $2^{++}$ & 6287.3 & 0.577 & & & \\
\hline \hline
\end{tabular}

TABLE IV. The eigenvectors of the $b b \bar{b} \bar{b}$ tetraquarks in the $b \bar{b} \otimes b \bar{b}$ configuration. The masses are all in units of $\mathrm{MeV}$.

\begin{tabular}{lcrrrrr}
\hline \hline System & $J^{P C}$ & Mass & $\Upsilon \Upsilon$ & $\Upsilon \eta_{b}$ & $\eta_{b} \Upsilon$ & $\eta_{b} \eta_{b}$ \\
\hline$b b \bar{b} \bar{b}$ & $0^{++}$ & 18836.1 & 0.514 & & & 0.584 \\
& & 18981.0 & -0.565 & & & 0.275 \\
& $1^{+-}$ & 18969.4 & & 0.408 & 0.408 & \\
& $2^{++}$ & 19000.1 & 0.577 & & & \\
\hline \hline
\end{tabular}

For simplicity, we only present the color-singlet components, and we rewrite the bases as a direct product of two mesons. For each decay mode, the branching fraction is proportional to the square of the coefficient $c_{i}$ of the corresponding component in the eigenvectors, and also depends on the phase space. For two body decay through $L$-wave, the partial decay width reads $[68,74]$

$$
\Gamma_{i}=\gamma_{i} \alpha \frac{k^{2 L+1}}{m^{2 L}} \cdot\left|c_{i}\right|^{2},
$$

where $\gamma_{i}$ is a quantity determined by the decay dynamics, $\alpha$ is an effective coupling constant, $m$ is the mass of the initial state, and $k$ is the momentum of the final states in the rest frame of the initial state. For the decays of the $S$-wave tetraquarks, $(\mathrm{k} / \mathrm{m})^{2}$ is of order $10^{-2}$ or even smaller. All higher wave decays are suppressed. Thus we will only consider the $S$-wave decays in this work. Employing the eigenvectors in Tables III-IV, we can calculate the value of $k \cdot\left|c_{i}\right|^{2}$ for each decay process (see Tables V-VI). Next we should consider the $\gamma_{i}$. Generally, $\gamma_{i}$ is determined by the spatial wave functions of both initial and final states, which are different for each decay process. In the quark model, the spatial wave functions of the pseudoscalar and vector mesons are the same. Thus for each tetraquark, we have

$$
\gamma_{\psi \psi}=\gamma_{\psi \eta_{c}}=\gamma_{\eta_{c} \eta_{c}}
$$

and

$$
\gamma_{\Upsilon \Upsilon}=\gamma_{\Upsilon_{\eta_{b}}}=\gamma_{\eta_{b} \eta_{b}}
$$

The values of the relative widths of different decay processes are listed in Tables VII and VIII. For the $c c \bar{c} \bar{c}$ tetraquarks, the ground state of the $0^{++}$is above the

TABLE V. The values of $k \cdot\left|c_{i}\right|^{2}$ for the $c c \bar{c} \bar{c}$ tetraquarks (in units of $\mathrm{MeV}$ ).

\begin{tabular}{lccccr}
\hline \hline System & $J^{P C}$ & Mass & $\psi \psi$ & $\psi \eta_{c}$ & $\eta_{c} \eta_{c}$ \\
\hline$c c \bar{c} \bar{c}$ & $0^{++}$ & 6044.9 & 0 & & 182.8 \\
& & 6271.3 & 195.8 & & 35.3 \\
& $1^{+-}$ & 6230.6 & & 226.3 & \\
& $2^{++}$ & 6287.3 & 180.1 & & \\
\hline \hline
\end{tabular}

TABLE VI. The values of $k \cdot\left|c_{i}\right|^{2}$ for the $b b \bar{b} \bar{b}$ tetraquarks (in units of $\mathrm{MeV}$ ).

\begin{tabular}{lccccr}
\hline \hline System & $J^{P C}$ & Mass & $\Upsilon \Upsilon$ & $\Upsilon \eta_{b}$ & $\eta_{b} \eta_{b}$ \\
\hline$b b \bar{b} \bar{b}$ & $0^{++}$ & 18836.1 & 0 & & 205.6 \\
& & 18981.0 & 241.5 & & 99.9 \\
& $1^{+-}$ & 18969.4 & & 340.6 & \\
& $2^{++}$ & 19000.1 & 289.4 & & \\
\hline \hline
\end{tabular}


TABLE VII. The partial width ratios for the $c c \bar{c} \bar{c}$ tetraquarks. For each state, we chose one mode as the reference channel, and the partial width ratios of the other channels are calculated relative to this channel. The masses are all in units of $\mathrm{MeV}$.

\begin{tabular}{lllllc}
\hline \hline System & $J^{P C}$ & Mass & $\psi \psi$ & $\psi \eta_{c}$ & $\eta_{c} \eta_{c}$ \\
\hline$c c \bar{c} \bar{c}$ & $0^{++}$ & 6044.9 & 0 & & 1 \\
& & 6271.3 & 5.6 & & 1 \\
& $1^{+-}$ & 6230.6 & & 1 & \\
& $2^{++}$ & 6287.3 & 1 & & \\
\hline \hline
\end{tabular}

TABLE VIII. The partial width ratios for the $b b \bar{b} \bar{b}$ tetraquarks. For each state, we chose one mode as the reference channel, and the partial width ratios of the other channels are calculated relative to this channel. The masses are all in units of $\mathrm{MeV}$.

\begin{tabular}{lcclcc}
\hline \hline System & $J^{P C}$ & Mass & $\Upsilon \Upsilon$ & $\Upsilon \eta_{b}$ & $\eta_{b} \eta_{b}$ \\
\hline$b b \bar{b} \bar{b}$ & $0^{++}$ & 18836.1 & 0 & & 1 \\
& & 18981.0 & 2.4 & & 1 \\
& $1^{+-}$ & 18969.4 & & 1 & \\
& $2^{++}$ & 19000.1 & 1 & & \\
\hline \hline
\end{tabular}

dissociation channel $\eta_{c} \eta_{c}$, thus it may be broad [62]. The other scalar state, $T\left(\operatorname{cc} \bar{c} \bar{c}, 6271.3,0^{++}\right)$, has two $S$-wave decay modes, namely $\psi \psi \psi$ and $\eta_{c} \eta_{c}$, their relative decay width ratio is

$$
\frac{\Gamma\left[T\left(c c \bar{c} \bar{c}, 6271.3,0^{++}\right) \rightarrow \psi \psi\right]}{\Gamma\left[T\left(c c \bar{c} \bar{c}, 6271.3,0^{++}\right) \rightarrow \eta_{c} \eta_{c}\right]}=5.6 .
$$

The dominant decay mode is the $\psi \psi$ final state. The $b b \bar{b} \bar{b}$ tetraquarks are very similar to the $c c \bar{c} \bar{c}$ tetraquarks. The ground state $T\left(b b \bar{b} \bar{b}, 18836.1,0^{++}\right)$can only decay into $\eta_{b} \eta_{b}$, while the higher scalar state $T\left(b b \bar{b} \bar{b}, 18981.0,0^{++}\right)$ can decay into both $\Upsilon \Upsilon$ and $\eta_{b} \eta_{b}$ channels, with relative decay with ratio

$$
\frac{\Gamma\left[T\left(b b \bar{b} \bar{b}, 18981.0,0^{++}\right) \rightarrow \Upsilon \Upsilon\right]}{\Gamma\left[T\left(b b \bar{b} \bar{b}, 18981.0,0^{++}\right) \rightarrow \eta_{b} \eta_{b}\right]}=2.4 .
$$

Thus the widths of the two modes do no differ very much.

\section{The $c c \bar{c} \bar{b}$ and $b b \bar{b} \bar{c}$ systems}

Next we consider the $c c \bar{c} \bar{b}$ and $b b \bar{b} \bar{c}$ tetraquarks. In Table IX, we list the masses and eigenvectors of these states. Their relative position and possible decay channels are plotted in Fig. 2. Since the $B_{c}^{*}$ has not been observed in experiment [4], we use the $M_{B_{c}^{*}}=6338 \mathrm{MeV}$ predicted by the Godfrey-Isgur model [58] to estimate the dimeson thresholds.

For these states, the two antiquarks do not have to obey the Pauli principle. We have three, rather than one, bases with $J^{P}=1^{+}$, which provides us a new platform to study
TABLE IX. Masses and eigenvectors of the $c c \bar{c} \bar{b}$ and $b b \bar{b} \bar{c}$ tetraquarks. The masses are all in units of $\mathrm{MeV}$.

\begin{tabular}{llcc}
\hline \hline System & $J^{P}$ & Mass & Eigenvector \\
\hline$c c \bar{c} \bar{b}$ & $0^{+}$ & 9317.5 & $\{0.869,0.495\}$ \\
& & 9505.9 & $\{-0.495,0.869\}$ \\
& $1^{+}$ & 9335.1 & $\{0.941,0.140,-0.306\}$ \\
& & 9484.3 & $\{0.190,-0.972,0.138\}$ \\
& & 9498.5 & $\{0.279,0.188,0.942\}$ \\
& $2^{+}$ & 9525.9 & $\{1\}$ \\
$b b \bar{b} \bar{c}$ & $0^{+}$ & 15711.9 & $\{0.908,0.418\}$ \\
& & 15862.0 & $\{-0.418,0.908\}$ \\
& $1^{+}$ & 15719.1 & $\{0.968,-0.005,-0.252\}$ \\
& & 15851.3 & $\{-0.015,-0.999,-0.037\}$ \\
& & 15854.4 & $\{-0.251,0.039,-0.967\}$ \\
& $2^{+}$ & 15882.3 & $\{1\}$ \\
\hline \hline
\end{tabular}

the color mixing. First we consider the $c c \bar{c} \bar{b}$ system. The ground state is $T\left(c c \bar{c} \bar{b}, 9317.5,0^{+}\right)$. This state is dominated by the color-sextet component $(75.5 \%)$, just as the $Q Q \bar{Q}^{\prime} \bar{Q}^{\prime}$ tetraquarks. The reason is similar to that in the $Q Q \bar{Q}^{\prime} \bar{Q}^{\prime}$ tetraquarks. It is interesting that the same mechanism can also apply to the $1^{+}$states. For the $c c \bar{c} \bar{b}$ tetraquarks, the color interaction reads

$$
\left\langle H_{\mathrm{C}}(c c \bar{c} \bar{b})\right\rangle=m_{c \bar{c}}+m_{c \bar{b}}-\frac{3}{2} \delta m^{\prime}\left\langle V_{12}^{\mathrm{C}}+V_{34}^{\mathrm{C}}\right\rangle
$$

where $\delta m^{\prime}=\left(3 \delta m_{b}+\delta m_{c}\right) / 4=42.89 \mathrm{MeV}$. There are three bases with $J^{P}=1^{+}$, namely $\left|(c c)_{0}^{6}(\bar{c} \bar{b})_{1}^{\overline{6}}\right\rangle$, $\left|(c c)_{1}^{\overline{3}}(\bar{c} \bar{b})_{1}^{3}\right\rangle$ and $\left|(c c)_{1}^{\overline{3}}(\bar{c} \bar{b})_{0}^{3}\right\rangle$ [see Eq. (16)]. Inserting these bases into the color interaction [Eq. (29)], we have

$$
\left\langle H_{\mathrm{C}}(c c \bar{c} \bar{b})\right\rangle=m_{c \bar{c}}+m_{c \bar{b}}+\delta m^{\prime}\left(\begin{array}{ccc}
-1 & 0 & 0 \\
0 & +2 & 0 \\
0 & 0 & +2
\end{array}\right) .
$$

We find that the color interaction does not mix these bases, just as in the $0^{+(+)}$cases. Moreover, the color interaction splits the three bases into two bands. The color-sextet base is more stable than the two color-triplet bases by 128.7 MeV. The CM interaction will further split the two color-triplet bases, and give the three-band structure in Fig. 2(a). The $b b \bar{b} \bar{c}$ tetraquarks are very similar to the $c c \bar{c} \bar{b}$ tetraquarks. Here we also find the ground state has $J^{P}=0^{+}$, which is dominated by the color-sextet $\left|(b b)_{0}^{6}(\bar{b} \bar{c})_{0}^{\overline{6}}\right\rangle$ component. For the $1^{+}$state, the lightest state $T\left(b b \bar{b} \bar{c}, 15719.1,1^{+}\right)$is mostly composed of $\left|(b b)_{0}^{6}(\bar{b} \bar{c})_{1}^{\overline{6}}\right\rangle(93.7 \%)$ bases, while the two higher states $T\left(b b \bar{b} \bar{c}, 15851.3,1^{+}\right)$and $T\left(b b \bar{b} \bar{c}, 15854.4,1^{+}\right)$are dominated by $\left|(b b)_{1}^{\overline{3}}(\bar{b} \bar{c})_{1}^{3}\right\rangle(99.8 \%)$ and $\left|(b b)_{1}^{\overline{3}}(\bar{b} \bar{c})_{0}^{3}\right\rangle(93.5 \%)$ components respectively. 


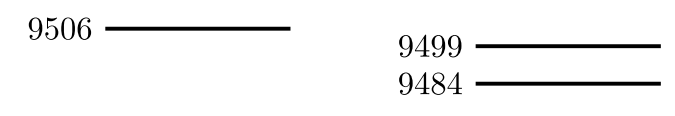

$\psi B_{c}^{*}$

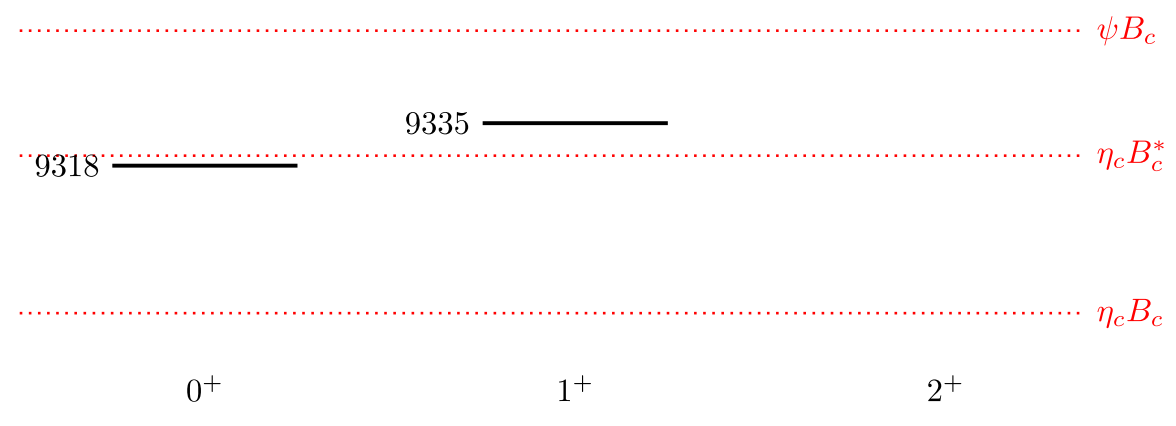

(a) $c c \bar{c} \bar{b}$ states

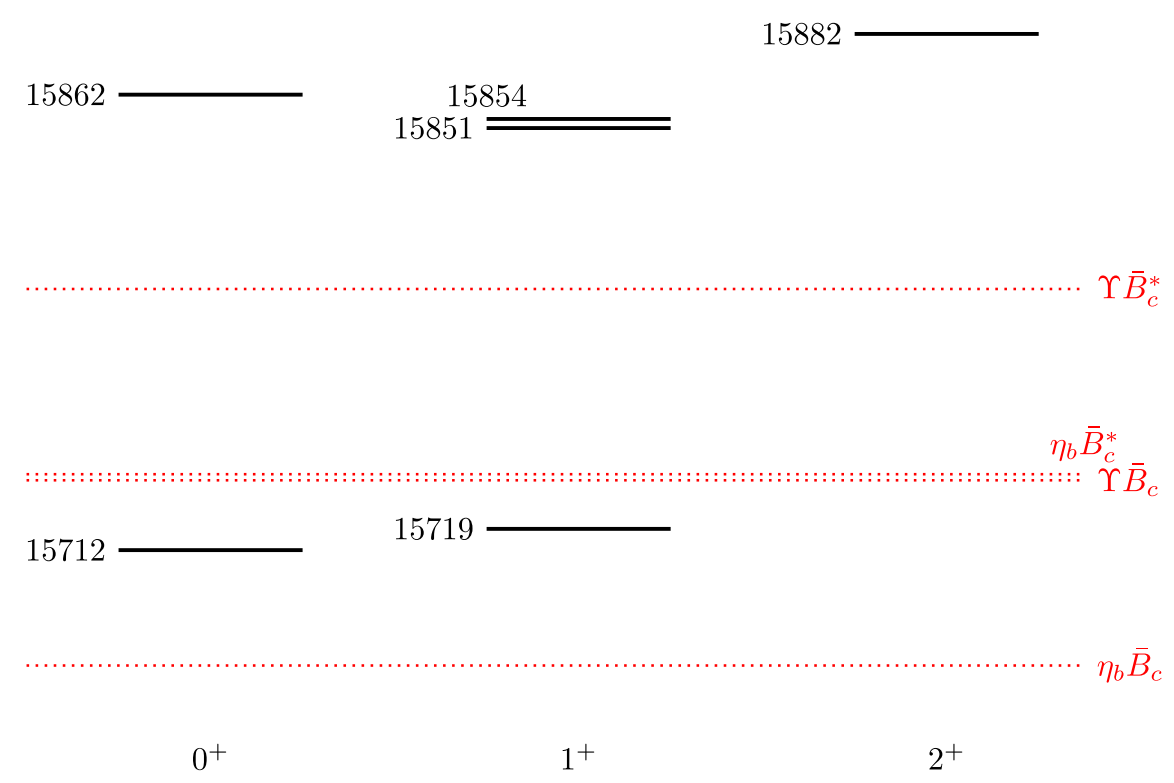

(b) $b b \bar{b} \bar{c}$ states

FIG. 2. Mass spectra of the $c c \bar{c} \bar{b}$ and $b b \bar{b} \bar{c}$ tetraquark states. The dotted lines indicate various meson-meson thresholds. Here the predicted mass $M_{B_{c}^{*}}=6338 \mathrm{MeV}$ of Godfrey et al. [58] is used. The masses are all in units of $\mathrm{MeV}$.

To study their decay properties, we transform the $c c \bar{c} \bar{b}$ $(b b \bar{b} \bar{c})$ tetraquarks into the $c \bar{c} \otimes c \bar{b}(b \bar{b} \otimes b \bar{c})$ configuration, and calculate their partial decay width ratios. The corresponding results can be found in Tables $\mathrm{X}-\mathrm{XV}$. From Table XV, we find that the $T\left(b b \bar{b} \bar{c}, 15719.1,1^{+}\right)$state does not have $S$-wave decay channel. Moreover, the $\eta_{b} \bar{B}_{c}$ channel is also forbidden because of the conservation of the angular momentum and parity. Thus we conclude that the $T\left(b b \bar{b} \bar{c}, 15719.1,1^{+}\right)$tetraquark is a narrow state.

One might wonder whether the conclusion will still hold if considering the following factors. Owing to the lack of experimental data, we use the predicted $B_{c}^{*}$ mass of Ref. [58] to estimate the dimeson thresholds. Moreover, the $T\left(b b \bar{b} \bar{c}, 15719.1,1^{+}\right)$state is only $16.1 \mathrm{MeV}(17.9 \mathrm{MeV})$ 
TABLE X. The eigenvectors of the $c c \bar{c} \bar{b}$ tetraquarks in the $c \bar{c} \otimes c \bar{b}$ configuration. The masses are all in units of $\mathrm{MeV}$.

\begin{tabular}{llrrrrr}
\hline \hline System & $J^{P}$ & Mass & $\psi B_{c}^{*}$ & $\psi B_{c}$ & $\eta_{c} B_{c}^{*}$ & $\eta_{c} B_{c}^{*}$ \\
\hline$c c \bar{c} \bar{b}$ & $0^{+}$ & 9317.5 & 0.472 & & & 0.602 \\
& & 9505.9 & -0.601 & & & 0.232 \\
& $1^{+}$ & 9335.1 & 0.418 & -0.416 & 0.530 & \\
& & 9484.3 & 0.166 & -0.435 & -0.359 & \\
& & 9498.5 & -0.545 & -0.235 & 0.081 & \\
& $2^{+}$ & 9525.9 & 0.577 & & & \\
\hline \hline
\end{tabular}

TABLE XI. The eigenvectors of the $b b \bar{b} \bar{c}$ tetraquarks in the $b \bar{b} \otimes b \bar{c}$ configuration. The masses are all in units of $\mathrm{MeV}$.

\begin{tabular}{lcrrrrr}
\hline \hline System & $J^{P}$ & Mass & $\Upsilon \bar{B}_{c}^{*}$ & $\Upsilon \bar{B}_{c}$ & $\eta_{b} \bar{B}_{c}^{*}$ & $\eta_{b} \bar{B}_{c}$ \\
\hline$b b \bar{b} \bar{c}$ & $0^{+}$ & 15711.9 & 0.521 & & & 0.580 \\
& & 15862.0 & -0.558 & & & 0.283 \\
& $1^{+}$ & 15719.1 & 0.456 & -0.470 & 0.466 & \\
& & 15851.3 & -0.023 & -0.413 & -0.403 & \\
& & 15854.4 & -0.540 & -0.161 & 0.193 & \\
& $2^{+}$ & 15882.3 & 0.577 & & & \\
\hline \hline
\end{tabular}

TABLE XII. The values of $k \cdot\left|c_{i}\right|^{2}$ for the $c c \bar{c} \bar{b}$ tetraquarks (in units of $\mathrm{MeV}$ ).

\begin{tabular}{lccccrr}
\hline \hline System & $J^{P}$ & Mass & $\psi B_{c}^{*}$ & $\psi B_{c}$ & $\eta_{c} B_{c}^{*}$ & $\eta_{c} B_{c}^{*}$ \\
\hline$c c \bar{c} \bar{b}$ & $0^{+}$ & 9317.5 & $\times$ & & & 177.2 \\
& & 9505.9 & 196.8 & & & 54.9 \\
& $1^{+}$ & 9335.1 & $\times$ & $\times$ & 65.1 & \\
& & 9484.3 & 12.5 & 129.6 & 105.4 & \\
& & 9498.5 & 153.4 & 40.2 & 5.7 & \\
& $2^{+}$ & 9525.9 & 205.9 & & & \\
\hline \hline
\end{tabular}

TABLE XIII. The values of $k \cdot\left|c_{i}\right|^{2}$ for the $b b \bar{b} \bar{c}$ tetraquarks (in units of $\mathrm{MeV}$ ).

\begin{tabular}{lcccccr}
\hline \hline System & $J^{P}$ & Mass & $\Upsilon \bar{B}_{c}^{*}$ & $\Upsilon \bar{B}_{c}$ & $\eta_{b} \bar{B}_{c}^{*}$ & $\eta_{b} \bar{B}_{c}$ \\
\hline$b b \bar{b} \bar{c}$ & $0^{+}$ & 15711.9 & $\times$ & & & 180.7 \\
& & 15862.0 & 216.8 & & & 95.9 \\
& $1^{+}$ & 15719.1 & $\times$ & $\times$ & $\times$ & \\
& & 15851.3 & 0.3 & 159.6 & 151.8 & \\
& & 15854.4 & 190.4 & 24.5 & 35.1 & \\
& $2^{+}$ & 15882.3 & 266.6 & & & \\
\hline \hline
\end{tabular}

below the $\Upsilon \bar{B}_{c}\left(\eta_{b} \bar{B}_{c}^{*}\right)$ threshold. The uncertainty of the present model may be large enough to push the state upward, then this state may have an $S$-wave decay channel. However, we note that the predicted $B_{c}^{*}$ mass was also used in parameter extraction, thus the error may be partially
TABLE XIV. The partial width ratios for the $c c \bar{c} \bar{b}$ tetraquarks. For each state, we chose one mode as the reference channel, and the partial width ratios of the other channels are calculated relative to this channel. The masses are all in units of $\mathrm{MeV}$.

\begin{tabular}{ccccccc}
\hline \hline System & $J^{P}$ & Mass & $\psi B_{c}^{*}$ & $\psi B_{c}$ & $\eta_{c} B_{c}^{*}$ & $\eta_{c} B_{c}^{*}$ \\
\hline$c c \bar{c} \bar{b}$ & $0^{+}$ & 9317.5 & $\times$ & & & 1 \\
& & 9505.9 & 3.6 & & & 1 \\
& $1^{+}$ & 9335.1 & $\times$ & $\times$ & 1 & \\
& & 9484.3 & 1 & 10.4 & 8.4 & \\
& & 9498.5 & 1 & 0.3 & 0.04 & \\
& $2^{+}$ & 9525.9 & 1 & & & \\
\hline \hline
\end{tabular}

TABLE XV. The partial width ratios for the $b b \bar{b} \bar{c}$ tetraquarks. For each state, we chose one mode as the reference channel, and the partial width ratios of the other channels are calculated relative to this channel. The masses are all in units of $\mathrm{MeV}$.

\begin{tabular}{llccccc}
\hline \hline System & $J^{P}$ & Mass & $\Upsilon \bar{B}_{c}^{*}$ & $\Upsilon \bar{B}_{c}$ & $\eta_{b} \bar{B}_{c}^{*}$ & $\eta_{b} \bar{B}_{c}$ \\
\hline$b b \bar{b} \bar{c}$ & $0^{+}$ & 15711.9 & $\times$ & & & 1 \\
& & 15862.0 & 2.3 & & & 1 \\
& $1^{+}$ & 15719.1 & $\times$ & $\times$ & $\times$ & \\
& & 15851.3 & 0.002 & 1.1 & 1 & \\
& & 15854.4 & 5.4 & 0.7 & 1 & \\
& $2^{+}$ & 15882.3 & 1 & & & \\
\hline \hline
\end{tabular}

cancelled with each other. Moreover, even if the mass of this state is pushed upward above the $\Upsilon \bar{B}_{c} / \eta_{b} \bar{B}_{c}^{*}$ threshold, its phase space is still relatively small. Then the decay width of this state is still relatively small compared to that of other states. We hope that future experiment can search for this state.

\section{The $c c \bar{b} \bar{b}$ system}

We list the numerical results of the $c c \bar{b} \bar{b}$ tetraquark in Table XVI. We also plot the mass spectrum and relevant meson-meson thresholds in Fig. 3. The pattern of mass spectrum is very similar to that of the $c c \bar{c} \bar{c} / b b \bar{b} \bar{b}$ tetraquarks. The ground state and most heavy state both have quantum number $0^{+}$. Their masses are $12596.3 \mathrm{MeV}$ and 12711.9 MeV respectively. The corresponding splitting is $115.6 \mathrm{MeV}$, which is smaller than that of both $c c \bar{c} \bar{c}$

TABLE XVI. Masses and eigenvectors of the $c c \bar{b} \bar{b}$ tetraquarks. The masses are all in units of $\mathrm{MeV}$.

\begin{tabular}{llcc}
\hline \hline System & $J^{P}$ & Mass & Eigenvector \\
\hline$c c \bar{b} \bar{b}$ & $0^{+}$ & 12596.3 & $\{0.718,0.696\}$ \\
& & 12711.9 & $\{-0.696,0.718\}$ \\
& $1^{+}$ & 12671.7 & $\{1\}$ \\
& $2^{+}$ & 12703.1 & $\{1\}$ \\
\hline \hline
\end{tabular}




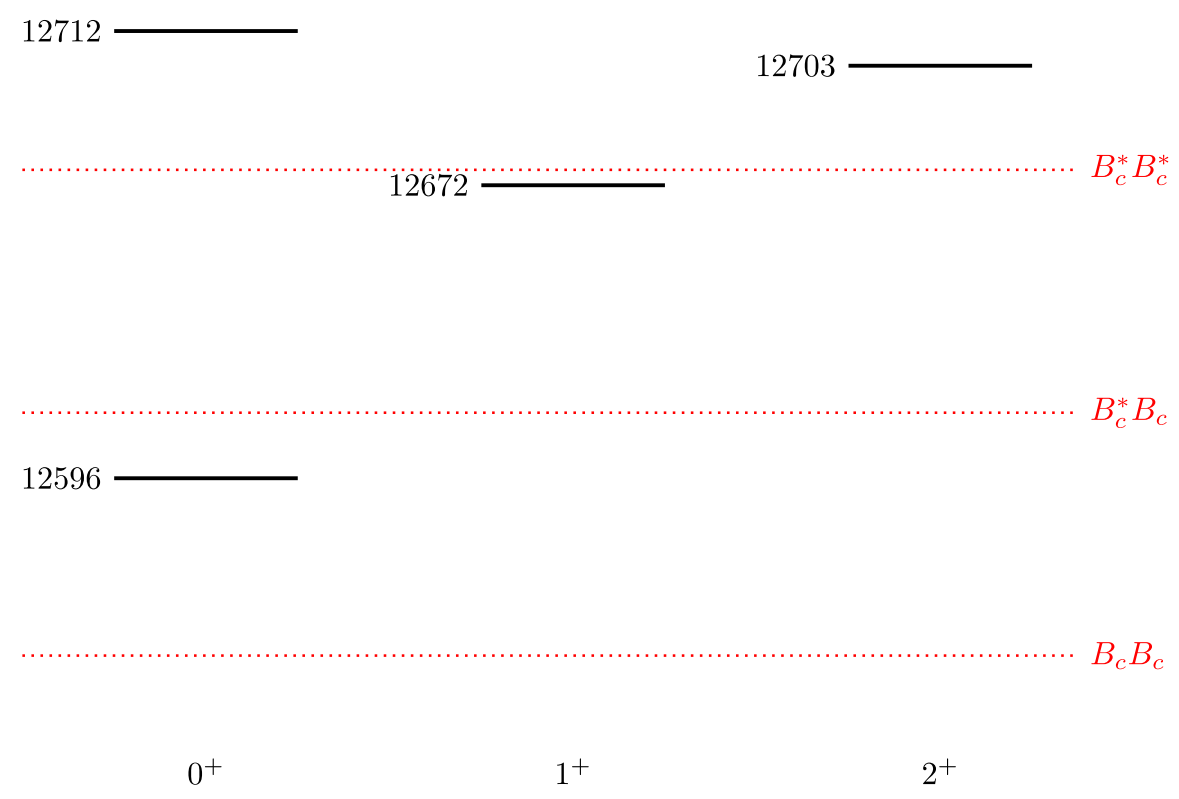

FIG. 3. Mass spectra of the $c c \bar{b} \bar{b}$ tetraquark states. The dotted lines indicate various meson-meson thresholds. Here the predicted mass $M_{B_{c}^{*}}=6338 \mathrm{MeV}$ of Godfrey et al. [58] is used. The masses are all in units of MeV.

$(226.4 \mathrm{MeV})$ and $b b \bar{b} \bar{b}(144.9 \mathrm{MeV})$ tetraquarks. The reason is as follows. With different quark/antiquark flavors, the color interaction becomes

$$
\left\langle H_{\mathrm{C}}(c c \bar{b} \bar{b})\right\rangle=2 m_{c \bar{b}}+\delta \tilde{m}\left(\begin{array}{cc}
-1 & 0 \\
0 & +2
\end{array}\right)
$$

where

$$
\delta \tilde{m}=\frac{1}{2}\left(\frac{m_{c c}+m_{b b}}{2}-m_{c \bar{b}}\right)=14.15 \mathrm{MeV}
$$

which is much smaller than the $\delta m_{Q}$ of Sec. III B. Thus the splitting becomes smaller. Another consequence is that the $\mathrm{CM}$ interaction becomes relatively more important, thus the $\overline{3}_{c} \otimes 3_{c}$ component becomes more important in the ground state. This can be seen from the wave function listed in Table XVI, where the $\overline{3}_{c} \otimes 3_{c}$ component of the ground state increases to $48.4 \%$. If we increase the mass difference between the quark and antiquark (such as $q q \bar{Q} \bar{Q}$ tetraquarks), the dominance of the $6_{c} \otimes \overline{6}_{c}$ or $\overline{3}_{c} \otimes 3_{c}$ component may be reversed [75]. Note that in some quark model studies, such reversing has already happened in the $c c \bar{b} \bar{b}$ system. In Ref. [42], Wang et al. studied the $Q Q \bar{Q}^{\prime} \bar{Q}^{\prime}$ tetraquark with two different quark model. They found that in the model II, the ground state has $53 \%$ of the $\overline{3}_{c} \otimes 3_{c}$ component, which is slightly larger than the $6_{c} \otimes \bar{\sigma}_{c}$ component. A detailed study of the dependence of the mass spectrum and wave function with respect to the mass ratio $m_{q} / m_{\bar{q}}$ is very important to reveal the nature of tetraquarks.
Next we consider their decay properties. We transform the wave functions of the $c c \bar{b} \bar{b}$ tetraquark into the $c \bar{b} \otimes c \bar{b}$ configuration, as shown in Table XVII. Experimentally, only the $B_{c}$ states have been found [4]. Here we use the $M_{B_{c}^{*}}=6338 \mathrm{MeV}$ predicted by the Godfrey-Isgur model [58], as we did in Ref. [67] to estimate the model parameters. The $k \cdot\left|c_{i}\right|^{2}$ values and relative decay widths are listed in Tables XVIII-XIX, where we have assumed

$$
\gamma_{B_{c}^{*} B_{c}^{*}}=\gamma_{B_{c}^{*} B_{c}}=\gamma_{B_{c} B_{c}^{*}}=\gamma_{B_{c} B_{c}} .
$$

TABLE XVII. The eigenvectors of the $c c \bar{b} \bar{b}$ tetraquarks in the $c \bar{b} \otimes c \bar{b}$ configuration. The masses are all in units of $\mathrm{MeV}$.

\begin{tabular}{lccrccc}
\hline \hline System & $J^{P}$ & Mass & $B_{c}^{*} B_{c}^{*}$ & $B_{c}^{*} B_{c}$ & $B_{c} B_{c}^{*}$ & $B_{c} B_{c}$ \\
\hline$c c \bar{b} \bar{b}$ & $0^{+}$ & 12596.3 & 0.307 & & & 0.641 \\
& & 12711.9 & -0.699 & & & 0.075 \\
& $1^{+}$ & 12671.7 & & 0.408 & 0.408 & \\
& $2^{+}$ & 12703.1 & 0.577 & & & \\
\hline \hline
\end{tabular}

TABLE XVIII. The values of $k \cdot\left|c_{i}\right|^{2}$ for the $c c \bar{b} \bar{b}$ tetraquarks (in units of $\mathrm{MeV}$ ).

\begin{tabular}{lccccr}
\hline \hline System & $J^{P C}$ & Mass & $B_{c}^{*} B_{c}^{*}$ & $B_{c}^{*} B_{c}+B_{c} B_{c}^{*}$ & $B_{c} B_{c}$ \\
\hline$c c \bar{b} \bar{b}$ & $0^{+}$ & 12596.3 & $\times$ & & 222.2 \\
& & 12711.9 & 233.5 & & 5.7 \\
& $1^{+}$ & 12671.7 & & 203.1 & \\
& $2^{+}$ & 12703.1 & 138.2 & & \\
\hline \hline
\end{tabular}


TABLE XIX. The partial width ratios for the $c c \bar{b} \bar{b}$ tetraquarks. For each state, we chose one mode as the reference channel, and the partial width ratios of the other channels are calculated relative to this channel. The masses are all in units of $\mathrm{MeV}$.

\begin{tabular}{cccccc}
\hline \hline System & $J^{P C}$ & Mass & $B_{c}^{*} B_{c}^{*}$ & $B_{c}^{*} B_{c}+B_{c} B_{c}^{*}$ & $B_{c} B_{c}$ \\
\hline$c c \bar{b} \bar{b}$ & $0^{+}$ & 12596.3 & $\times$ & & 1 \\
& & 12711.9 & 41.0 & & 1 \\
& $1^{+}$ & 12671.7 & & 1 & \\
& $2^{+}$ & 12703.1 & 1 & & \\
\hline \hline
\end{tabular}

Similar to the $c c \bar{c} \bar{c} / b b \bar{b} \bar{b}$ cases, we find that all states are above the thresholds into two $S$-wave mesons. Thus they are broad [62]. The ground state can only decay into two $B_{c}$ mesons. And the other scalar state $T\left(c c \bar{b} \bar{b}, 12711.9,0^{+}\right)$ decays into both $B_{c} B_{c}$ and $B_{c}^{*} B_{c}^{*}$ modes. Their partial decay width ratio is

$$
\frac{\Gamma\left[T\left(c c \bar{b} \bar{b}, 12711.9,0^{++}\right) \rightarrow B_{c}^{*} B_{c}^{*}\right]}{\Gamma\left[T\left(c c \bar{b} \bar{b}, 12711.9,0^{++}\right) \rightarrow B_{c} B_{c}\right]}=41.0
$$

Thus the $B_{c}^{*} B_{c}^{*}$ mode is dominant. The other two states $T\left(c c \bar{b} \bar{b}, 12671.7,1^{+}\right)$and $T\left(c c \bar{b} \bar{b}, 12703.1,2^{+}\right)$can decay into $B_{c} B_{c}^{*}$ and $B_{c}^{*} B_{c}^{*}$ modes respectively in $S$-wave.

\section{E. The $c b \bar{c} \bar{b}$ system}

Now we turn to the $c b \bar{c} \bar{b}$ system. The mass spectra and eigenvectors are listed in Table XX. We also transform the eigenvectors into the $c \bar{c} \otimes b \bar{b}$ and $c \bar{b} \otimes b \bar{c}$ configurations, as shown in Tables. XXI-XXII. From Table XXI, we see that the eigenvector of the lowest state reads

$$
T\left(c b \bar{c} \bar{b}, 12362.8,0^{++}\right)=0.960 \eta_{c} \eta_{c}+\cdots .
$$

This state couples very strongly to the $\eta_{c} \eta_{c}$ channel. It is broad and is just a part of the continuum. Note that this kind
TABLE XXI. The eigenvectors of the $c b \bar{c} \bar{b}$ tetraquarks in the

\begin{tabular}{|c|c|c|c|c|c|c|}
\hline System & $J^{P C}$ & Mass & $\psi \Upsilon$ & $\psi \eta_{b}$ & $\eta_{c} \Upsilon$ & $\eta_{c} \eta_{b}$ \\
\hline \multirow{12}{*}{$c b \bar{c} \bar{b}$} & $0^{++}$ & 12362.8 & -0.097 & & & 0.960 \\
\hline & & 12509.3 & 0.840 & & & 0.200 \\
\hline & & 12681.6 & 0.529 & & & -0.123 \\
\hline & & 12746.9 & -0.066 & & & 0.152 \\
\hline & $1^{++}$ & 12523.6 & -0.902 & & & \\
\hline & & 12703.2 & 0.433 & & & \\
\hline & $1^{+-}$ & 12424.9 & & 0.174 & 0.948 & \\
\hline & & 12477.2 & & 0.942 & -0.215 & \\
\hline & & 12720.0 & & -0.261 & -0.198 & \\
\hline & & 12744.1 & & 0.117 & -0.124 & \\
\hline & $2^{++}$ & 12537.4 & 0.953 & & & \\
\hline & & 12754.9 & 0.302 & & & \\
\hline
\end{tabular}
$c_{1} \bar{c}_{3} \otimes b_{2} \bar{b}_{4}$ configuration. The masses are all in units of $\mathrm{MeV}$.

TABLE XXII. The eigenvectors of the $c b \bar{c} \bar{b}$ tetraquarks in the $c_{1} \bar{b}_{4} \otimes b_{2} \bar{c}_{3}$ configuration. The masses are all in units of $\mathrm{MeV}$.

\begin{tabular}{lcrrrrr}
\hline \hline System & $J^{P C}$ & Mass & $B_{c}^{*} \bar{B}_{c}^{*}$ & $B_{c}^{*} \bar{B}_{c}$ & $B_{c} \bar{B}_{c}^{*}$ & $B_{c} \bar{B}_{c}$ \\
\hline$c b \bar{c} \bar{b}$ & $0^{++}$ & 12362.8 & -0.348 & & & 0.420 \\
& & 12509.3 & -0.302 & & & -0.673 \\
& & 12681.6 & 0.208 & & & 0.574 \\
& & 12746.9 & 0.863 & & & -0.205 \\
& $1^{++}$ & 12523.6 & & 0.501 & -0.501 & \\
& & 12703.2 & & 0.499 & -0.499 & \\
& $1^{+-}$ & 12424.9 & 0.259 & 0.355 & 0.355 & \\
& & 12477.2 & -0.418 & 0.259 & 0.259 & \\
& & 12720.0 & -0.041 & 0.552 & 0.552 & \\
& 12744.1 & 0.870 & 0.044 & 0.044 & \\
& 2 & 12537.4 & 0.602 & & & \\
& & 12754.9 & -0.798 & & & \\
\hline \hline
\end{tabular}

\begin{tabular}{|c|c|c|c|c|}
\hline System & $J^{P C}$ & Mass & Eigenvector & Scattering state \\
\hline \multirow[t]{12}{*}{$c b \bar{c} \bar{b}$} & $0^{++}$ & 12362.8 & $\{0.868,0.298,0.296,0.264\}$ & \multirow{4}{*}{$\eta_{c} \eta_{b}$} \\
\hline & & 12509.3 & $\{-0.415,0.873,0.160,0.199\}$ & \\
\hline & & 12681.6 & $\{0.013,-0.043,-0.662,0.748\}$ & \\
\hline & & 12746.9 & $\{-0.272,-0.383,0.670,0.575\}$ & \\
\hline & $1^{++}$ & 12523.6 & $\{0.697,0.697,0.118,0.118\}$ & \multirow[t]{2}{*}{$\psi \Upsilon$} \\
\hline & & 12703.2 & $\{-0.118,-0.118,0.697,0.697\}$ & \\
\hline & $1^{+-}$ & 12424.9 & $\{0.794,-0.349,0.349,0.252,-0.176,0.176\}$ & \multirow{4}{*}{$\begin{array}{l}\eta_{c} \Upsilon \\
\psi \eta_{b}\end{array}$} \\
\hline & & 12477.2 & $\{0.539,0.535,-0.535,0.128,0.245,-0.245\}$ & \\
\hline & & 12720.0 & $\{0.279,-0.002,0.002,-0.957,-0.053,0.053\}$ & \\
\hline & & 12744.1 & $\{0.035,-0.303,0.303,-0.058,0.637,-0.637\}$ & \\
\hline & $2^{++}$ & 12537.4 & $\{0.953,0.304\}$ & \multirow[t]{2}{*}{$\psi \Upsilon$} \\
\hline & & 12754.9 & $\{-0.304,0.953\}$ & \\
\hline
\end{tabular}

TABLE XX. Masses and eigenvectors of the $c b \bar{c} \bar{b}$ tetraquarks. The masses are all in units of MeV. 


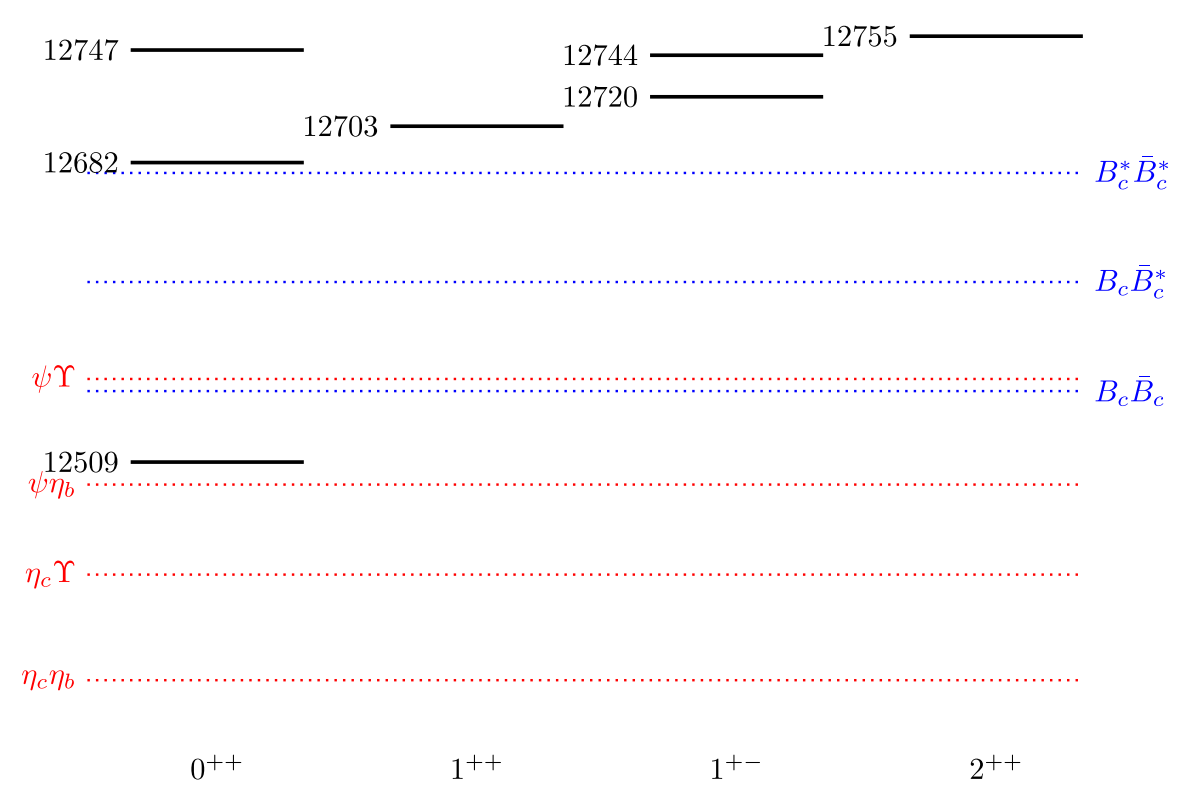

FIG. 4. Mass spectra of the $c b \bar{c} \bar{b}$ tetraquark states. The dotted lines indicate various meson-meson thresholds. Here the predicted mass $M_{B_{c}^{*}}=6338 \mathrm{MeV}$ of Godfrey et al. [58] is used. The masses are all in units of MeV.

of state also exists in our previous study of the hidden charm pentaquark, where some of the calculated states couple strongly to a charmonium and a light baryons [68]. Similar phenomenon has also been found in $c \bar{c} q \bar{q}$ tetraquark $[12,69]$. Moreover, the states of $12523.6 \mathrm{MeV}$ (with $J^{P C}=1^{++}$) and $12537.4 \mathrm{MeV}$ (with $J^{P C}=2^{++}$) couple strongly to $\psi$ and $\Upsilon$ mesons. They are also scattering states. The remaining coupling type of $\psi \otimes \Upsilon$ is $0^{++}$, which largely resides in $T\left(c b \bar{c} \bar{b}, 12509.3,0^{++}\right)$. However, this state possesses large fractions (25\%) of the color-octet components, thus we cannot rule out the possibility that it is a tetraquark. To draw a more definitive conclusion, we need to study its internal dynamics $[42,76,77]$, which is beyond the present work. There are two additional scattering states composed of a vector meson and a pseudoscalar meson. The $T\left(c b \bar{c} \bar{b}, 12424.9,1^{+-}\right)$is a scattering state of $\eta_{c} \Upsilon$ and $T\left(c b \bar{c} \bar{b}, 12477.2,1^{+-}\right)$is a scattering state of $\psi \eta_{b}$. For clarity, we add a fifth column in Table XX to indicate these scattering states.

In Fig. 4, we present the relative position of the $c b \bar{c} \bar{b}$ tetraquarks. We also plot all the relevant meson-meson thresholds. To study their decay properties, we need to estimate the $\gamma_{i}$. In the quark model, the spatial wave functions of the ground state scalar and vector meson are the same. Thus for each $c b \bar{c} \bar{b}$ tetraquark

$$
\gamma_{\psi \Upsilon}=\gamma_{\psi \eta_{b}}=\gamma_{\eta_{c} \Upsilon}=\gamma_{\eta_{c} \eta_{b}}
$$

and

$$
\gamma_{B_{c}^{*} B_{c}^{*}}=\gamma_{B_{c}^{*} B_{c}}=\gamma_{B_{c} B_{c}^{*}}=\gamma_{B_{c} B_{c}} .
$$

Combining the eigenvectors in the $c \bar{c} \otimes b \bar{b}$ and $c \bar{b} \otimes b \bar{c}$ configurations, we can calculate the relative partial widths of different decay modes, as listed in Tables XXIII-XXIV.

The lowest $c b \bar{c} \bar{b}$ tetraquarks is $T\left(c b \bar{c} \bar{b}, 12509.3,0^{++}\right)$. This state can decay into $\eta_{c} \eta_{b}$ through $S$-wave. The $T\left(c b \bar{c} \bar{b}, 12681.6,0^{++}\right)$and $T\left(c b \bar{c} \bar{b}, 12746.9,0^{++}\right)$tetraquarks have the same decay channels. But their relative sizes of partial width are different, which can be used to distinguish the two states. More precisely, for $T\left(c b \bar{c} \bar{b}, 12681.6,0^{++}\right)$, we have

$$
\Gamma_{\psi \Upsilon}: \Gamma_{\eta_{c} \eta_{b}}=12.0,
$$

and

$$
\Gamma_{B_{c}^{*} \bar{B}_{c}^{*}}: \Gamma_{B_{c} \bar{B}_{c}}=0.03
$$

TABLE XXIII. The partial width ratios for the $c b \bar{c} \bar{b}$ tetraquarks decay into a charmonium plus a bottomonium. For each state, we chose one mode as the reference channel, and the partial width ratios of the other channels are calculated relative to this channel. The masses are all in units of $\mathrm{MeV}$.

\begin{tabular}{lcccccc}
\hline \hline System & $J^{P C}$ & Mass & $\psi \Upsilon$ & $\psi \eta_{b}$ & $\eta_{c} \Upsilon$ & $\eta_{c} \eta_{b}$ \\
\hline$c b \bar{c} \bar{b}$ & $0^{++}$ & 12509.3 & $\times$ & & & 1 \\
& & 12681.6 & 12.0 & & & 1 \\
& & 12746.9 & 0.1 & & & 1 \\
& $1^{++}$ & 12703.2 & 1 & & & \\
& $1^{+-}$ & 12720.0 & & 1.6 & 1 & \\
& & 12744.1 & & 0.8 & 1 & \\
& $2^{++}$ & 12754.9 & 1 & & & \\
\hline \hline
\end{tabular}


TABLE XXIV. The partial width ratios for the $c b \bar{c} \bar{b}$ tetraquarks decays into $B_{c}^{(*)} \bar{B}_{c}^{(*)}$. For each state, we chose one mode as the reference channel, and the partial width ratios of the other channels are calculated relative to this channel. The masses are all in units of $\mathrm{MeV}$.

\begin{tabular}{lcccccc}
\hline \hline System & $J^{P C}$ & Mass & $B_{c}^{*} \bar{B}_{c}^{*}$ & $B_{c}^{*} \bar{B}_{c}$ & $B_{c} \bar{B}_{c}^{*}$ & $B_{c} \bar{B}_{c}$ \\
\hline$c b \bar{c} \bar{b}$ & $0^{++}$ & 12509.3 & $\times$ & & & $\times$ \\
& & 12681.6 & 0.03 & & & 1 \\
& & 12746.9 & 10.7 & & & 1 \\
& $1^{++}$ & 12703.2 & & 1 & 1 & \\
& $1^{+-}$ & 12720.0 & 0.003 & 1 & 1 & \\
& & 12744.1 & 278.7 & 1 & 1 & \\
& $2^{++}$ & 12754.9 & 1 & & & \\
\hline \hline
\end{tabular}

And the $T\left(c b \bar{c} \bar{b}, 12746.9,0^{++}\right)$has

$$
\Gamma_{\psi \Upsilon}: \Gamma_{\eta_{c} \eta_{b}}=0.1,
$$

and

$$
\Gamma_{B_{c}^{*} \bar{B}_{c}^{*}}: \Gamma_{B_{c} \bar{B}_{c}}=10.7 \text {. }
$$

The other states (with $J=1$ and $J=2$ ) are all above the $B_{c}^{*} \bar{B}_{c}^{*}$ threshold. They may be broad since they can freely decay into many channels in $S$-wave.

\section{CONCLUSIONS}

In this work, we have systematically studied the mass spectrum of the fully heavy tetraquark in an extended chromomagnetic model, which includes both colorelectric and chromomagnetic interactions. There is no stable state below the lowest heavy quarkonium pair thresholds.
Most states can dissociate into two $S$-wave mesons through $S$-wave decay. Thus they may be wide states. One possible narrow state is the $1^{+} b b \bar{b} \bar{c}$ state with mass $15719.1 \mathrm{MeV}$. Although it is above the $\eta_{b} \bar{B}_{c}$ threshold, this channel is forbidden because of the conservation of the angular momentum and parity.

There are two possible color configurations of tetraquark, namely the color-sextet $\left|(Q Q)^{6_{c}}(\bar{Q} \bar{Q})^{\overline{6}_{c}}\right\rangle$ and the colortriplet $\left|(Q Q)^{\overline{3}_{c}}(\bar{Q} \bar{Q})^{3_{c}}\right\rangle$. From the eigenvectors obtained, we find that the energy level is mainly determined by the colorelectric interaction. The colorelectric interaction always favors the color-sextet configurations. Note that if $Q_{1}=Q_{2}$ or $\bar{Q}_{3}=\bar{Q}_{4}$, the colorelectric interaction does not mix the two color configurations. The chromomagnetic interaction favors the color-triplet configurations. But its contribution is relatively smaller than that of the colorelectric interaction, thus it only gives small splitting to the states. The chromomagnetic interaction can also mix the color-sextet and the color-triplet configurations. Comparing the ground states $\left[J^{P C}=0^{+(+)}\right]$of the $c c \bar{c} \bar{c} / b b \bar{b} \bar{b}$ and $c c \bar{b} \bar{b}$ tetraquarks, we find that the mixing becomes larger when there is a mass difference between the quark and antiquark. In other words, the color-triplet component is more important in the ground state $c c \bar{b} \bar{b}$ tetraquark than in the ground state $c c \bar{c} \bar{c} / b b \bar{b} \bar{b}$ tetraquarks.

With the eigenvectors obtained, we have also investigated the decay properties of the tetraquarks. We hope that future experiments can search for these states.

\section{ACKNOWLEDGMENTS}

X.Z.W. is grateful to G.J. Wang and L. Meng for helpful comments and discussions. This project is supported by the National Natural Science Foundation of China under Grants No. 11975033.
[1] M. Gell-Mann, A schematic model of baryons and mesons, Phys. Lett. 8, 214 (1964).

[2] G. Zweig, An $\mathrm{SU}_{3}$ model for strong interaction symmetry and its breaking; Version 1 (1964), http://cds.cern.ch/record/ 352337.

[3] S. K. Choi et al. (Belle Collaboration), Observation of a Narrow Charmoniumlike State in Exclusive $B^{ \pm} \rightarrow$ $K^{ \pm} \pi^{+} \pi^{-} J / \psi$ Decays, Phys. Rev. Lett. 91, 262001 (2003).

[4] P. A. Zyla et al. (Particle Data Group), Review of particle physics, Prog. Theor. Exp. Phys. 083C01 (2020).

[5] B. Aubert et al. (BABAR Collaboration), Observation of a Broad Structure in the $\pi^{+} \pi^{-} J / \psi$ Mass Spectrum around 4.26 GeV/c², Phys. Rev. Lett. 95, 142001 (2005).
[6] M. Ablikim et al. (BESIII Collaboration), Observation of a Charged Charmoniumlike Structure in $e^{+} e^{-} \rightarrow \pi^{+} \pi^{-} J / \psi$ at $\sqrt{s}=4.26 \mathrm{GeV}$, Phys. Rev. Lett. 110, 252001 (2013).

[7] Z. Q. Liu et al. (Belle Collaboration), Study of $e^{+} e^{-} \rightarrow$ $\pi^{+} \pi^{-} J / \psi$ and Observation of a Charged Charmoniumlike State at Belle, Phys. Rev. Lett. 110, 252002 (2013); Erratum, Phys. Rev. Lett. 111, 019901 (2013).

[8] A. Bondar et al. (Belle Collaboration), Observation of Two Charged Bottomoniumlike Resonances in $\Upsilon(5 S)$ Decays, Phys. Rev. Lett. 108, 122001 (2012).

[9] E. S. Swanson, Short range structure in the $X(3872)$, Phys. Lett. B 588, 189 (2004).

[10] T. F. Caramés, A. Valcarce, and J. Vijande, Charged charmonium molecules, Phys. Rev. D 82, 054032 (2010). 
[11] R. Chen, X. Liu, Y.-R. Liu, and S.-L. Zhu, Predictions of the hidden-charm molecular states with the four quark components, Eur. Phys. J. C 76, 319 (2016).

[12] Y. Cui, X.-L. Chen, W.-Z. Deng, and S.-L. Zhu, Possible heavy tetraquarks $q Q \bar{q} \bar{Q}, q q \bar{Q} \bar{Q}$ and $q Q \bar{Q} \bar{Q}$, High Energy Phys. Nucl. Phys. 31, 7 (2007) [arXiv:hep-ph/0607226].

[13] W. Park and S. H. Lee, Color spin wave functions of heavy tetraquark states, Nucl. Phys. A925, 161 (2014).

[14] S.-L. Zhu, The possible interpretations of $Y(4260)$, Phys. Lett. B 625, 212 (2005).

[15] A. Esposito, A. Pilloni, and A. D. Polosa, Hybridized tetraquarks, Phys. Lett. B 758, 292 (2016).

[16] H.-X. Chen, W. Chen, X. Liu, and S.-L. Zhu, The hiddencharm pentaquark and tetraquark states, Phys. Rep. 639, 1 (2016).

[17] A. Esposito, A. Pilloni, and A. D. Polosa, Multiquark resonances, Phys. Rep. 668, 1 (2017).

[18] R. F. Lebed, R. E. Mitchell, and E. S. Swanson, Heavyquark QCD exotica, Prog. Part. Nucl. Phys. 93, 143 (2017).

[19] A. Ali, J. S. Lange, and S. Stone, Exotics: Heavy pentaquarks and tetraquarks, Prog. Part. Nucl. Phys. 97, 123 (2017).

[20] F.-K. Guo, C. Hanhart, U.-G. Meißner, Q. Wang, Q. Zhao, and B.-S. Zou, Hadronic molecules, Rev. Mod. Phys. 90, 015004 (2018).

[21] C.-Z. Yuan, The XYZ states revisited, Int. J. Mod. Phys. A 33, 1830018 (2018).

[22] N. Brambilla, S. Eidelman, C. Hanhart, A. Nefediev, C.-P. Shen, C. E. Thomas, A. Vairo, and C.-Z. Yuan, The XYZ states: Experimental and theoretical status and perspectives, Phys. Rep. 873, 1 (2020).

[23] Y.-R. Liu, H.-X. Chen, W. Chen, X. Liu, and S.-L. Zhu, Pentaquark and tetraquark states, Prog. Part. Nucl. Phys. 107, 237 (2019).

[24] V. Khachatryan et al. (CMS Collaboration), Observation of $\Upsilon(1 S)$ pair production in proton-proton collisions at $\sqrt{s}=8 \mathrm{TeV}$, J. High Energy Phys. 05 (2017) 013.

[25] S. Durgut (CMS Collaboration), Search for exotic mesons at CMS (2018), https://meetings.aps.org/Meeting/APR18/ Session/U09.6.

[26] R. Aaij et al. (LHCb Collaboration), Search for beautiful tetraquarks in the $\Upsilon(1 S) \mu^{+} \mu^{-}$invariant-mass spectrum, J. High Energy Phys. 10 (2018) 086.

[27] L. C. Bland et al. ( $\mathrm{A}_{N} \mathrm{DY}$ Collaboration), Observation of Feynman scaling violations and evidence for a new resonance at RHIC, arXiv:1909.03124.

[28] J. Badier et al. (NA3 Collaboration), Evidence for $\psi \psi$ production in $\pi^{-}$interactions at 150 and $280 \mathrm{GeV} / c$, Phys. Lett. 114B, 457 (1982).

[29] J. Badier et al. (NA3 Collaboration), $\psi \psi \psi$ production and limits on beauty meson production from $400 \mathrm{GeV} / c$ protons, Phys. Lett. 158B, 85 (1985).

[30] V. M. Abazov et al. (D0 Collaboration), Observation and studies of double $J / \psi$ production at the Tevatron, Phys. Rev. D 90, 111101 (2014).

[31] R. Aaij et al. (LHCb Collaboration), Observation of charmonium pairs produced exclusively in $p p$ collisions, J. Phys. G 41, 115002 (2014).

[32] S. D. Yang et al. (Belle Collaboration), Evidence of $\Upsilon(1 S) \rightarrow J / \psi+\chi_{c 1}$ and search for double-charmonium production in $\Upsilon(1 S)$ and $\Upsilon(2 S)$ decays, Phys. Rev. D 90, 112008 (2014).

[33] R. Aaij et al. (LHCb Collaboration), Observation of structure in the $J / \psi$-pair mass spectrum, Sci. Bull. 65, 1983 (2020).

[34] Y. Bai, S. Lu, and J. Osborne, Beauty-full tetraquarks, Phys. Lett. B 798, 134930 (2019).

[35] M. Karliner, S. Nussinov, and J. L. Rosner, $Q Q \bar{Q} \bar{Q}$ states: Masses, production, and decays, Phys. Rev. D 95, 034011 (2017).

[36] J. Wu, Y.-R. Liu, K. Chen, X. Liu, and S.-L. Zhu, Heavyflavored tetraquark states with the $Q Q \bar{Q} \bar{Q}$ configuration, Phys. Rev. D 97, 094015 (2018).

[37] V. R. Debastiani and F. S. Navarra, A non-relativistic model for the $[c c][\bar{c} \bar{c}]$ tetraquark, Chin. Phys. C 43, 013105 (2019).

[38] J.-M. Richard, A. Valcarce, and J. Vijande, String dynamics and metastability of all-heavy tetraquarks, Phys. Rev. D 95, 054019 (2017).

[39] A. Esposito and A. D. Polosa, A $b b \bar{b} \bar{b}$ di-bottomonium at the LHC?, Eur. Phys. J. C 78, 782 (2018).

[40] M. A. Bedolla, J. Ferretti, C. D. Roberts, and E. Santopinto, Spectrum of fully-heavy tetraquarks from a diquark + antidiquark perspective, Eur. Phys. J. C 80, 1004 (2020).

[41] M.-S. Liu, Q.-F. Lü, X.-H. Zhong, and Q. Zhao, All-heavy tetraquarks, Phys. Rev. D 100, 016006 (2019).

[42] G.-J. Wang, L. Meng, and S.-L. Zhu, Spectrum of the fullyheavy tetraquark state $Q Q \bar{Q}^{\prime} \bar{Q}^{\prime}$, Phys. Rev. D 100, 096013 (2019).

[43] E. Braaten, L.-P. He, and A. Mohapatra, Masses of doubly heavy tetraquarks with error bars, Phys. Rev. D 103, 016001 (2021).

[44] C. Deng, H. Chen, and J. Ping, Towards the understanding of fully-heavy tetraquark states from various models, Phys. Rev. D 103, 014001 (2021).

[45] J. F. Giron and R. F. Lebed, Simple spectrum of $c \bar{c} c \bar{c}$ states in the dynamical diquark model, Phys. Rev. D 102, 074003 (2020).

[46] X. Jin, Y. Xue, H. Huang, and J. Ping, Full-heavy tetraquarks in constituent quark models, Eur. Phys. J. C 80, 1083 (2020).

[47] Q.-F. Lü, D.-Y. Chen, and Y.-B. Dong, Masses of fully heavy tetraquarks $Q Q \bar{Q} \bar{Q}$ in an extended relativized quark model, Eur. Phys. J. C 80, 871 (2020).

[48] W. Chen, H.-X. Chen, X. Liu, T. G. Steele, and S.-L. Zhu, Hunting for exotic doubly hidden-charm/bottom tetraquark states, Phys. Lett. B 773, 247 (2017).

[49] Z.-G. Wang, Analysis of the $Q Q \bar{Q} \bar{Q}$ tetraquark states with QCD sum rules, Eur. Phys. J. C 77, 432 (2017).

[50] W. Chen, H.-X. Chen, X. Liu, T. G. Steele, and S.-L. Zhu, Doubly hidden-charm/bottom $Q Q \bar{Q} \bar{Q}$ tetraquark states, EPJ Web Conf. 182, 02028 (2018).

[51] Z.-G. Wang and Z.-Y. Di, Analysis of the vector and axialvector $Q Q \bar{Q} \bar{Q}$ tetraquark states with $\mathrm{QCD}$ sum rules, Acta Phys. Pol. B 50, 1335 (2019).

[52] H.-X. Chen, W. Chen, X. Liu, and S.-L. Zhu, Strong decays of fully-charm tetraquarks into di-charmonia, Sci. Bull. 65, 1994 (2020).

[53] W. Heupel, G. Eichmann, and C. S. Fischer, Tetraquark bound states in a Bethe-Salpeter approach, Phys. Lett. B 718, 545 (2012). 
[54] C. Hughes, E. Eichten, and C. T. H. Davies, Searching for beauty-fully bound tetraquarks using lattice nonrelativistic QCD, Phys. Rev. D 97, 054505 (2018).

[55] M. N. Anwar, J. Ferretti, F.-K. Guo, E. Santopinto, and B.-S. Zou, Spectroscopy and decays of the fully-heavy tetraquarks, Eur. Phys. J. C 78, 647 (2018).

[56] E. Eichten, K. Gottfried, T. Kinoshita, K. D. Lane, and T.-M. Yan, Charmonium: The model, Phys. Rev. D 17, 3090 (1978); Erratum, Phys. Rev. D 21, 313 (1980).

[57] N. Isgur and G. Karl, Hyperfine interactions in negative parity baryons, Phys. Lett. 72B, 109 (1977).

[58] S. Godfrey and N. Isgur, Mesons in a relativized quark model with chromodynamics, Phys. Rev. D 32, 189 (1985).

[59] S. Capstick and N. Isgur, Baryons in a relativized quark model with chromodynamics, Phys. Rev. D 34, 2809 (1986); AIP Conf. Proc. 132, 267 (1985).

[60] A. De Rújula, H. Georgi, and S. L. Glashow, Hadron masses in a gauge theory, Phys. Rev. D 12, 147 (1975).

[61] Y. B. Zeldovich and A. D. Sakharov, Quark structure and masses of strongly interacting particles, Yad. Fiz. 4, 395 (1966) [Sov. J. Nucl. Phys. 4, 283 (1967)], https://www .osti.gov/biblio/4529076-quark-structure-masses-stronglyinteracting-particles.

[62] R. L. Jaffe, Multiquark hadrons. I. The phenomenology of $Q^{2} \bar{Q}^{2}$ mesons, Phys. Rev. D 15, 267 (1977).

[63] R. L. Jaffe, Multiquark hadrons. II. Methods, Phys. Rev. D 15, 281 (1977).

[64] F. Buccella, H. Høgaasen, J.-M. Richard, and P. Sorba, Chromomagnetism, flavour symmetry breaking and $S$-wave tetraquarks, Eur. Phys. J. C 49, 743 (2007).

[65] M. Karliner and J. L. Rosner, Baryons with two heavy quarks: Masses, production, decays, and detection, Phys. Rev. D 90, 094007 (2014).
[66] R. Aaij et al. (LHCb Collaboration), Observation of the Doubly Charmed Baryon $\Xi_{c c}^{++}$, Phys. Rev. Lett. 119, 112001 (2017).

[67] X.-Z. Weng, X.-L. Chen, and W.-Z. Deng, Masses of doubly heavy-quark baryons in an extended chromomagnetic model, Phys. Rev. D 97, 054008 (2018).

[68] X.-Z. Weng, X.-L. Chen, W.-Z. Deng, and S.-L. Zhu, Hidden-charm pentaquarks and $P_{c}$ states, Phys. Rev. D 100, 016014 (2019).

[69] H. Høgaasen, E. Kou, J.-M. Richard, and P. Sorba, Isovector and hidden-beauty partners of the $X(3872)$, Phys. Lett. B 732, 97 (2014).

[70] R. Aaij et al. (LHCb Collaboration), Observation of a Narrow Pentaquark State, $P_{c}(4312)^{+}$, and of the Two-Peak Structure of the $P_{c}(4450)^{+}$, Phys. Rev. Lett. 122, 222001 (2019).

[71] R. Aaij et al. (LHCb Collaboration), Observation of $J / \psi p$ Resonances Consistent with Pentaquark States in $\Lambda_{b}^{0} \rightarrow$ $J / \psi K^{-} p$ Decays, Phys. Rev. Lett. 115, 072001 (2015).

[72] B. Keren-Zur, Testing confining potentials through meson/ baryon hyperfine splittings, Ann. Phys. (Amsterdam) 323, 631 (2008).

[73] D. Strottman, Multiquark baryons and the MIT bag model, Phys. Rev. D 20, 748 (1979).

[74] C. Gao, Group Theory and its Application in Particle Physics (in Chinese) (Higher Education Press, Beijing, 1992).

[75] X.-Z. Weng et al. (to be published).

[76] T. Myo and K. Kato, Complex scaling: Physics of unbound light nuclei and perspective, Prog. Theor. Exp. Phys. 12A101 (2020).

[77] G. Yang, J. Ping, and J. Segovia, Tetra- and penta-quark structures in the constituent quark model, Symmetry 12, 1869 (2020). 